\title{
STRONG LOCAL SURVIVAL OF BRANCHING RANDOM WALKS IS NOT MONOTONE
}

\author{
DANIELA BERTACCHI,* Università di Milano-Bicocca \\ FABIO ZUCCA, ${ }^{* *}$ Politecnico di Milano
}

\begin{abstract}
In this paper we study the strong local survival property for discrete-time and continuoustime branching random walks. We study this property by means of an infinite-dimensional generating function $G$ and a maximum principle which, we prove, is satisfied by every fixed point of $G$. We give results for the existence of a strong local survival regime and we prove that, unlike local and global survival, in continuous time, strong local survival is not a monotone property in the general case (though it is monotone if the branching random walk is quasitransitive). We provide an example of an irreducible branching random walk where the strong local property depends on the starting site of the process. By means of other counterexamples, we show that the existence of a pure global phase is not equivalent to nonamenability of the process, and that even an irreducible branching random walk with the same branching law at each site may exhibit nonstrong local survival. Finally, we show that the generating function of an irreducible branching random walk can have more than two fixed points; this disproves a previously known result.
\end{abstract}

Keywords: Branching random walk; branching process; strong local survival; recurrence; generating function; maximum principle

2010 Mathematics Subject Classification: Primary 60J05

Secondary $60 \mathrm{~J} 80$

\section{Introduction}

A branching process is a very simple population model (introduced in [14]) where particles breed and die (independently of each other) according to some random law. At any time, this process is completely characterized by the total number of particles alive. Branching random walks (BRWs) add space to this picture: particles live in a spatially structured environment, and the reproduction law, which may depend on the location, not only tells us how many children the particle has, but also where it places them. The state of the process, at any time, is thus described by the collection of the numbers of particles alive at $x$, where $x$ varies among the possible sites. In the literature one can find BRWs both in continuous and discrete time. The continuous-time setting has been studied by many authors (see, e.g. [17], [18], [19], [20], and [22]) along with some variants of the process (see [2], [3], [4], [5], and [7]). The discrete-time case has been initially considered as a natural generalization of branching processes (see [1], [10], [11], [12], [13], and [16]). The definition of a discrete-time branching random walk (BRW) that we give in Section 2.1 is sufficiently general to include the discrete-time counterpart that every continuoustime BRW admits. Since every continuous-time BRW and its discrete-time counterpart have the

Received 4 September 2012; revision received 6 June 2013.

* Postal address: Dipartimento di Matematica e Applicazioni, Università di Milano-Bicocca, via Cozzi 53, 20125

Milano, Italy. Email address: daniela.bertacchi@unimib.it

** Postal address: Dipartimento di Matematica, Politecnico di Milano, Piazza Leonardo da Vinci 32, 20133 Milano, Italy. Email address: fabio.zucca@polimi.it 
same asymptotic behavior, it suffices to provide results for the discrete-time case. On the other hand, continuous-time examples naturally yield discrete-time examples. Our definition also includes the following as particular cases: BRWs with independent diffusion (where particles are first generated and then dispersed independently according to a diffusion matrix $P$; see Section 2.1 and (2.2)), BRWs with no death (where each particle has null probability of having no children), and BRWs whose total number of particles behaves as a branching process (where the law of the number of offspring does not depend on the site-we call these BRWs locally isomorphic to a branching process-see Section 2.4).

The basic questions which arise when studying the BRW are whether it survives with positive probability and, in this case, if it visits a given site infinitely many times. The first question addresses whether there is global survival, that is, with positive probability, at any time there is someone alive somewhere). The second question deals with local survival, that is, whether, with positive probability, the process returns infinitely many times to some fixed sites. It is clear that the probability of global survival is greater than or equal to the probability of local survival. If the probability of global survival is strictly greater than the probability of local survival then the latter may be positive or null. In the first case we say that there is nonstrong local survival, in the second case we say that there is pure global survival. When, on the contrary, the probabilities of global and local survival are equal and strictly positive, we say that the BRW has strong local survival. Hence, strong local survival means that the events of local and global survival coincide (but for a null probability set) and have positive probability.

The interest in the strong local behavior is fairly recent (see, for instance, [15] and [23]). Our aim is to study some properties of strong local survival, comparing them with the corresponding properties of local and global survival.

As in the case of branching processes, the main tool is that probabilities of extinction are fixed points of an infinite-dimensional generating function $G$ (see Section 2.3 for the definition and Section 3.2 for a link to the extinction probabilities). It is worth noting that, unlike the case of a branching process with finite types, in general, $G$ may have more than two fixed points, even in the irreducible case (where, with positive probability, a particle at site $x$ can have progenies at site $y$ for all $x$ and $y$ ). Indeed, we prove this indirectly by providing examples of irreducible BRWs which survive locally and globally but with different probabilities (hence, nonstrong locally; see Examples 4.4 and 4.5), and directly by an explicit construction of three fixed points for the $G$ of a certain BRW (see Remark 4.1). By Corollary 3.1, in the irreducible case, a sufficient condition for the existence of at most two fixed points for $G$ is the finiteness of the set of vertices.

In the particular case where there is no branching, we obtain a random walk, and the role of $G$ and its fixed points are respectively played by the transition matrix and the harmonic functions. It is thus natural to look for a maximum principle in the context of BRWs as well (see Proposition 2.1). As an application, in the irreducible case, pure global survival is independent of the starting vertex. This is also true for local and global survival, but it does not hold for strong local survival, unless the probability of having zero children is positive for all sites or if the BRW is quasitransitive (see Sections 2.4 and 3.2 and Corollary 3.2). Example 4.3 shows that we may have strong local survival starting from some vertices and nonstrong local survival starting from others.

The speed of reproduction of a continuous-time BRW is proportional to a positive parameter $\lambda$ (see Section 2.2). It is easily seen that the probabilities of local and global survival are nondecreasing functions with respect to $\lambda$; thus, local and global survival are monotone properties (meaning that if one of them holds for some $\lambda_{0}$ then it holds for all $\lambda \geq \lambda_{0}$ ), 
and it is possible to define the local and global critical parameters $\lambda_{s}$ and $\lambda_{w}$ (see Section 2.2). We show that monotonicity in $\lambda$ does not hold for strong local survival and it is thus impossible, in general, to define a strong local critical parameter: for the irreducible BRW in Example 4.2, if $\lambda$ is small enough or large enough, there is strong local survival, but in an intermediate interval for $\lambda$ there is global and local survival with different probabilities.

The paper is organized as follows. In Section 2 we give the necessary definitions and some basic facts about discrete-time BRWs (Section 2.1), continuous-time BRWs (Section 2.2), the infinite-dimensional generating function $G$, defined on $[0,1]^{X}$ associated to a BRW (Section 2.3), and the special class of $\mathcal{F}$-BRWs (Section 2.4). This class properly contains the class of BRWs on quasitransitive graphs (which were studied in [26]). We also give explicit expressions for $G$ in the particular cases of a BRW with independent diffusion (see (2.4)) and a BRW with no death, constructed from a BRW with death, disregarding all particles with finite progenies (see (2.5)). Moreover, in Section 2.3 we state a maximum principle for the solutions of the equation $G(v) \geq v$, including all fixed points of $G$ (Proposition 2.1).

Section 3 is devoted to the study of all the types of survival. We first recall, in Section 3.1, results on local and global survival (Theorems 3.1 and 3.2). In Section 3.2 extinction probabilities are seen as fixed points of the generating function $G$. Theorem 3.3 gives equivalent conditions for strong local survival, in terms of extinction probabilities, which are useful to prove that strong local survival is not monotone.

From the maximum principle we derive Theorem 3.4, which describes some properties of fixed points of $G$ for $\mathcal{F}$-BRWs, and Corollaries 3.1 and 3.2. Corollary 3.2 shows that, for an irreducible, quasitransitive BRW, there are only three possible behaviors (independently of the starting vertex): global extinction, pure global survival, or strong local survival. Thus, in this case, strong local survival is monotone and the critical parameter is $\lambda_{s}$. A characterization of strong local survival in terms of the existence of a solution of some inequalities involving the generating function $G$ is given by Theorem 3.5.

Section 4 is devoted to examples and counterexamples. For a continuous-time irreducible $\mathcal{F}$-BRW, the existence of a pure global phase is equivalent to nonamenability (see Section 2.1 and Section 3.3). Nevertheless, in general, nonamenability neither implies nor is implied by the existence of a pure global phase (Example 4.1). Finally, we show (Examples 4.4 and 4.5) that even fairly simple BRWs (such as irreducible BRWs with independent diffusion and with offspring distribution independent of the site) may have nonstrong local survival (see also Example 4.2). This implies that, even in the irreducible case, the generating function $G$ may have more than two fixed points in $[0,1]^{X}$ and disproves a result in [25] (see Remark 4.1).

\section{Basic definitions and preliminaries}

\subsection{Discrete-time BRWs}

We start with the construction of a generic discrete-time BRW $\left\{\eta_{n}\right\}_{n \in \mathbb{N}}$ (see also [8], where it is called an infinite-type branching process) on a set $X$ which is at most countable; $\eta_{n}(x)$ represents the number of particles alive at $x \in X$ at time $n$. To this end, we consider a family $\mu=\left\{\mu_{x}\right\}_{x \in X}$ of probability measures on the (countable) measurable space $\left(S_{X}, 2^{S_{X}}\right)$, where $S_{X}:=\left\{f: X \rightarrow \mathbb{N}: \sum_{y} f(y)<\infty\right\}$. To obtain generation $n+1$ from generation $n$, we proceed as follows: a particle at site $x \in X$ lives for one unit of time, then a function $f \in S_{X}$ is chosen at random according to the law $\mu_{x}$ and the original particle is replaced by $f(y)$ particles at $y$ for all $y \in X$; this is done independently for all particles of generation $n$ (for a similar construction in a random environment, see [15]). Note that the choice of $f$ simultaneously 
assigns the total number of children and the location where they will live. We denote the BRW by the couple $(X, \mu)$.

Equivalently, we could introduce the BRW by first choosing the number of children and then their location. Define $\mathscr{H}: S_{X} \rightarrow \mathbb{N}$ as $\mathscr{H}(f):=\sum_{y \in X} f(y)$, which represents the total number of children associated to $f$. Denote by $\rho_{x}$ the measure on $\mathbb{N}$ defined by $\rho_{x}(\cdot):=\mu_{x}\left(\mathscr{H}^{-1}(\cdot)\right)$; this is the law of the random number of children of a particle living at $x$. For each particle, independently, we pick a number $n$ at random, according to the law $\rho_{x}$, then we choose a function $f \in \mathscr{H}^{-1}(n)$ with probability $\mu_{x}(f) / \rho_{x}(n) \equiv \mu_{x}(f) / \sum_{g \in \mathscr{H}^{-1}(n)} \mu_{x}(g)$, and we replace the particle at $x$ with $f(y)$ particles at $y$ (for all $y \in X$ ).

In BRW theory, a fundamental role is played by the first-moment matrix $M=\left(m_{x y}\right)_{x, y \in X}$, where $m_{x y}:=\sum_{f \in S_{X}} f(y) \mu_{x}(f)$ is the expected number of particles from $x$ to $y$ (that is, the expected number of children that a particle living at $x$ sends to $y$ ). We suppose that $\sup _{x \in X} \sum_{y \in X} m_{x y}<+\infty$. Most of the results of this paper still hold without this hypothesis; nevertheless, it allows us to avoid dealing with an infinite expected number of offspring. The expected number of children generated by a particle living at $x$ is

$$
\sum_{y \in X} m_{x y}=\sum_{n \geq 0} n \rho_{x}(n)=: \bar{\rho}_{x} .
$$

Given a function $f$ defined on $X$, we denote by $M f$ the function $M f(x):=\sum_{y \in X} m_{x y} f(y)$ whenever the right-hand side converges absolutely for all $x$. We denote by $m_{x y}^{(n)}$ the entries of the $n$th power matrix $M^{n}$, and we define

$$
M_{s}(x, y):=\limsup _{n \rightarrow \infty} \sqrt[n]{m_{x y}^{(n)}}, \quad M_{w}(x):=\liminf _{n \rightarrow \infty} \sqrt[n]{\sum_{y \in X} m_{x y}^{(n)}} \text { for all } x, y \in X .
$$

Explicit computations of $M_{S}(x, y)$ and $M_{w}(x)$ are possible in some cases (see [6] and [8]). In particular, $M_{s}(x, x)$ can be obtained by means of a generating function (see [28, Section 3.2]). In this paper we do not need to explicitly compute $M_{s}$ and $M_{w}$, except for some specific examples where justifications will be provided.

For a generic BRW, we define the diffusion matrix as the matrix $P$ with entries $p(x, y)=$ $m_{x y} / \bar{\rho}_{x}$. In particular, if $\bar{\rho}_{x}$ does not depend on $x \in X$, we have $M_{w}(x)=\bar{\rho}$ for all $x \in X$ and $M_{S}(x, y)=\bar{\rho} \lim \sup _{n \rightarrow \infty} \sqrt[n]{p^{(n)}(x, y)}$ (where the lim sup defines the spectral radius of $P$ according to [27, Chapter I, Section 1.B]).

Note that, in the general case, the locations of the offspring are not chosen independently (they are assigned by the chosen $f \in S_{X}$ ). When the offspring are dispersed independently according to $P$ we call the process a $B R W$ with independent diffusion; in this case

$$
\mu_{x}(f)=\rho_{x}\left(\sum_{y} f(y)\right) \frac{\left(\sum_{y} f(y)\right) !}{\prod_{y} f(y) !} \prod_{y} p(x, y)^{f(y)} \quad \text { for all } f \in S_{X} .
$$

To a generic discrete-time BRW we associate a graph $\left(X, E_{\mu}\right)$, where $(x, y) \in E_{\mu}$ if and only if $m_{x y}>0$. We denote by $\operatorname{deg}(x)$ the degree of a vertex $x$, that is, the cardinality of the set $\mathcal{N}_{x}:=\left\{y \in X:(x, y) \in E_{\mu}\right\}$. We say that there is a path from $x$ to $y$, writing $x \rightarrow y$, if it is possible to find a finite sequence $\left\{x_{i}\right\}_{i=0}^{n}$ (where $n \in \mathbb{N}$ ) such that $x_{0}=x, x_{n}=y$, and $\left(x_{i}, x_{i+1}\right) \in E_{\mu}$ for all $i=0, \ldots, n-1$. If $x \rightarrow y$ and $y \rightarrow x$, we write $x \rightleftharpoons y$. Observe that there is always a path of length 0 from $x$ to itself. The equivalence class $[x]$ of $x$ with respect 
to ' $\rightleftharpoons$ ' is called an irreducible class of $x$. It is easy to show that if $x \rightleftharpoons x^{\prime}$ and $y \rightleftharpoons y^{\prime}$, then $M_{S}(x, y)=M_{s}\left(x^{\prime}, y^{\prime}\right)$ and $M_{w}(x)=M_{w}\left(x^{\prime}\right)$. Moreover, $m_{x x}^{(n)}$ and $M_{s}(x, x)$ depend only on

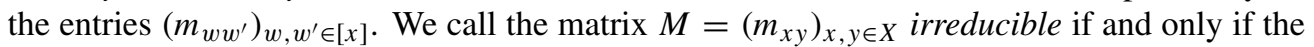
graph $\left(X, E_{\mu}\right)$ is connected (that is, there is only one irreducible class); otherwise, we call it reducible. From the BRW point of view, the irreducibility of $M$ means that the progeny of any particle can spread to any site of the graph. For an irreducible BRW, $M_{s}(x, y)=M_{s}$ and $M_{w}(x)=M_{w}$ for all $x, y \in X$.

The BRW $(X, \mu)$ is called nonoriented or symmetric if $m_{x y}=m_{y x}$ for every $x, y \in X$. Note that if $(X, \mu)$ is nonoriented then the graph $\left(X, E_{\mu}\right)$ is nonoriented (that is, $(x, y) \in E_{\mu}$ if and only if $\left.(y, x) \in E_{\mu}\right)$. We call $(X, \mu)$ nonamenable if and only if

$$
\inf \left\{\frac{\sum_{x \in S, y \in S^{\complement}} m_{x y}}{|S|}: S \subseteq X,|S|<\infty\right\}>0,
$$

and we call it amenable otherwise.

The idea behind the definition of nonamenability is that the expected number of children placed outside every finite subset of $X$ is always comparable with the size of the subset itself. This suggests that, in principle, it should be possible for the BRW to survive and, at the same time, to escape from every finite set. This is true for a subclass of BRWs, but not in general; see Example 4.1 and the preceding discussion. We note that if $m_{x y} \in\{0, \lambda\}$ (for some fixed $\lambda>0$ ) then the BRW is nonamenable if and only if the graph $\left(X, E_{\mu}\right)$ is nonamenable according to the usual definition for graphs (see [27, Chapter II, Section 12.B]).

Depending on the initial configuration, the process can survive in different ways. We consider initial configurations with only one particle placed at a fixed site $x$ : let $\mathbb{P}^{\delta_{x}}$ be the law of this process. Throughout this paper, 'w.p.p.ís shorthand for 'with positive probability'.

Definition 2.1. (a) The process survives locally w.p.p. in $A \subseteq X$ starting from $x \in X$ if $\boldsymbol{q}(x, A):=1-\mathbb{P}^{\delta_{x}}\left(\lim \sup _{n \rightarrow \infty} \sum_{y \in A} \eta_{n}(y)>0\right)<1$.

(b) The process survives globally w.p.p. starting from $x$ if $\overline{\boldsymbol{q}}(x):=\boldsymbol{q}(x, X)<1$.

(c) There is strong local survival w.p.p. in $A \subseteq X$ starting from $x \in X$ if $\boldsymbol{q}(x, A)=\overline{\boldsymbol{q}}(x)<1$ and nonstrong local survival w.p.p. in $A$ if $\overline{\boldsymbol{q}}(x)<\boldsymbol{q}(x, A)<1$.

(d) The BRW is in a pure global survival phase starting from $x$ if $\overline{\boldsymbol{q}}(x)<\boldsymbol{q}(x, x)=1$ (where we write $\boldsymbol{q}(x, y)$ instead of $\boldsymbol{q}(x,\{y\})$ for all $x, y \in X)$.

From now on, when we talk about survival, 'w.p.p.will be tacitly understood. Often we will say simply that local survival occurs 'starting from $x$ ' or 'at $x$ '; in this case we mean that $x=y$. When there is no survival w.p.p., we say that there is extinction, and the fact that extinction occurs with probability 1 will be tacitly understood.

Note that $\boldsymbol{q}(x, A)$ are the probabilities of extinction in $A$ starting from $x$. Roughly speaking, there is strong survival at $y$ starting from $x$ if and only if the probability of local survival at $y$ starting from $x$, conditioned on global survival starting from $x$, is 1 . Thus, strong local survival means that, for almost all realizations, the process either survives locally (hence globally) or it goes globally extinct. There are many relations between $\overline{\boldsymbol{q}}(x)$ and $\boldsymbol{q}(x, y)$ and between $\boldsymbol{q}(w, x)$ and $\boldsymbol{q}(w, y)$, where $x, y, w \in X$ (see, for instance, Section 3.2, [9] or [28]).

In order to avoid trivial situations where particles have one offspring almost surely, we henceforth make the following assumption. 
Assumption 2.1. For all $x \in X$, there is a vertex $y \rightleftharpoons x$ such that

$$
\mu_{y}\left(f: \sum_{\{w: w \rightleftharpoons y\}} f(w)=1\right)<1,
$$

that is, in every equivalence class (with respect to ' $\rightleftharpoons$ ') there is at least one vertex where a particle can have inside the class a number of children different from 1 w.p.p.

\subsection{Continuous-time BRWs}

In continuous time, each particle has an exponentially distributed random lifetime with parameter 1 . The breeding mechanisms can be regulated by means of a nonnegative matrix $K=\left(k_{x y}\right)_{x, y \in X}$ in such a way that, for each particle alive at $x$, there is a clock with $\operatorname{Exp}\left(\lambda k_{x y}\right)$ distributed intervals (where $\lambda>0$ ), and each time the clock rings the particle places one son at $y$. We say that the BRW has a death rate 1 and a reproduction rate $\lambda k_{x y}$ from $x$ to $y$. We observe (see Remark 2.1) that the assumption of a nonconstant death rate does not represent a significant generalization. We denote by $(X, K)$ a family of continuous-time BRWs (depending on the parameter $\lambda>0)$, while we use the notation $(X, \mu)$ for a discrete-time BRW.

To a continuous-time BRW one can associate a discrete-time counterpart which takes into account all the offspring of a particle before it dies; in this sense the theory of continuous-time BRWs, as long as it concerns the probabilities of survival (local, strong local, and global), is a particular case of the theory of discrete-time BRWs. Elementary calculations show that $\mu_{x}$ satisfies (2.2), where

$$
\rho_{x}(i)=\frac{1}{1+\lambda k(x)}\left(\frac{\lambda k(x)}{1+\lambda k(x)}\right)^{i}, \quad p(x, y)=\frac{k_{x y}}{k(x)}
$$

$\left(k(x):=\sum_{y \in X} k_{x y}\right)$. Note that the discrete-time counterpart of a continuous-time BRW is a BRW with independent diffusion and that $\rho_{x}$ depends only on $\lambda k(x)$. It is straightforward to show that $m_{x y}=\lambda k_{x y}$ and $\bar{\rho}_{x}=\lambda k(x)$. Moreover, (2.3) shows that the discrete-time counterpart satisfies Assumption 2.1. All the definitions given in the discrete-time case extend to continuous-time BRWs: a continuous-time BRW has some property if and only if its discretetime counterpart has it.

Remark 2.1. The same construction applies to continuous-time BRWs with a death rate $d(x)>0$ dependent on $x \in X$. In this case, the discrete-time counterpart satisfies (2.2), where

$$
\rho_{x}(i)=\frac{d(x)}{d(x)+\lambda k(x)}\left(\frac{\lambda k(x)}{d(x)+\lambda k(x)}\right)^{i}, \quad p(x, y)=\frac{k_{x y}}{k(x)} .
$$

Hence, from the point of view of local and global survival, this process is equivalent to a continuous-time BRW with death rate 1 and reproduction rate $\lambda k_{x y} / d(x)$ from $x$ to $y$.

Given $x \in X$, two critical parameters are associated to the continuous-time BRW: the global survival critical parameter $\lambda_{w}(x)$ and the local survival critical parameter $\lambda_{s}(x)$. They are defined as

$$
\begin{aligned}
& \lambda_{w}(x):=\inf \left\{\lambda>0: \mathbb{P}^{\delta_{x}}\left(\sum_{w \in X} \eta_{t}(w)>0 \text { for all } t\right)>0\right\}, \\
& \lambda_{s}(x):=\inf \left\{\lambda>0: \mathbb{P}^{\delta_{x}}\left(\limsup _{t \rightarrow \infty} \eta_{t}(x)>0\right)>0\right\} .
\end{aligned}
$$

These values are constant in every irreducible class; in particular, they do not depend on $x$ if the BRW is irreducible. The process is called globally supercritical, critical, or subcritical if 
$\lambda>\lambda_{w}, \lambda=\lambda_{w}$, or $\lambda<\lambda_{w}$; an analogous definition is given for the local behavior using $\lambda_{s}$ instead of $\lambda_{w}$. In particular, we say that there exists a pure global survival phase starting from $x$ if the interval $\left(\lambda_{w}(x), \lambda_{s}(x)\right)$ is not empty; clearly, if $\lambda \in\left(\lambda_{w}(x), \lambda_{s}(x)\right)$ then the BRW is in a pure global survival phase according to Definition 2.1 .

Given a continuous-time BRW $(X, K)$, we define, for all $x, y \in X$,

$$
K_{s}(x, y):=\frac{M_{s}(x, y)}{\lambda} \equiv \limsup _{n \rightarrow \infty} \sqrt[n]{k_{x y}^{(n)}}, \quad K_{w}(x):=\frac{M_{w}(x)}{\lambda} \equiv \liminf _{n \rightarrow \infty} \sqrt[n]{\sum_{y \in X} k_{x y}^{(n)}},
$$

where $M_{s}(x, y)$ and $M_{w}(x)$ are the corresponding parameters of the discrete-time counterpart. Here $K_{s}(x, y)$ and $K_{w}(x)$ depend only on the equivalence classes of $x$ and $y$; hence, if the BRW is irreducible then they do not depend on $x, y \in X$.

We say that a BRW is site breeding if $k(x)$ does not depend on $x \in X$. We say that a $\mathrm{BRW}$ is edge breeding if $k_{x y} \in \mathbb{N}$. The typical edge-breeding BRW can be constructed from a multigraph with a set of vertices $X$ by defining $k_{x y}$ as the number of edges from $x$ to $y$; in this case to each edge there corresponds a constant reproduction rate $\lambda$. If the multigraph is a graph then it coincides with the graph $\left(X, E_{\mu}\right)$ associated with the discrete-time counterpart of the edge-breeding BRW.

\subsection{Infinite-dimensional generating function}

We associate a generating function $G:[0,1]^{X} \rightarrow[0,1]^{X}$ to the family $\left\{\mu_{x}\right\}_{x \in X}$, which can be considered as an infinite-dimensional power series. More precisely, for all $z \in[0,1]^{X}$, $G(z) \in[0,1]^{X}$ is defined as the following weighted sum of (finite) products:

$$
G(z \mid x):=\sum_{f \in S_{X}} \mu_{x}(f) \prod_{y \in X} z(y)^{f(y)} .
$$

Here $G(z \mid x)$ is the $x$ coordinate of $G(z)$. The family $\left\{\mu_{x}\right\}_{x \in X}$ is uniquely determined by $G$. Indeed, fix a finite $X_{0} \subseteq X$ and $x \in X$. For every $z$ with support in $X_{0}$, we have $G(z \mid x)=\sum_{f \in S_{X_{0}}} \mu_{x}(f) \prod_{y \in X_{0}} z(y)^{f(y)}$, which can be identified with a power series with several variables (defined on $[0,1]^{X_{0}}$ ). Suppose now that we have another generating function $\bar{G}$ (associated to $\left\{\bar{\mu}_{x}\right\}_{x \in X}$ ) such that $G=\bar{G}$. In particular, $G(z \mid x)=\bar{G}(z \mid x)$ for every $z$ with support in $X_{0}$. Thus, $\mu_{x}(f)=\bar{\mu}_{x}(f)$ for all $f \in S_{X_{0}}$. Since $S_{X}=\bigcup_{\left\{X_{0} \subseteq X: X_{0} \text { finite }\right\}} S_{X_{0}}$, we have $\mu_{x}(f)=\bar{\mu}_{x}(f)$ for all $f \in S_{X}$.

Note that $G$ is continuous with respect to the pointwise convergence topology of $[0,1]^{X}$ and nondecreasing with respect to the usual partial order of $[0,1]^{X}$ (see [8, Sections 2 and 3$]$ for further details). Moreover, $G$ represents the 1-step reproductions; we denote by $G^{(n)}$ the generating function associated to the $n$-step reproductions, which is inductively defined as $G^{(n+1)}(z)=G^{(n)}(G(z))$, where $G^{(0)}$ is the identity. Extinction probabilities are fixed points of $G$ and the smallest fixed point is $\overline{\boldsymbol{q}}$ (see Section 3.2 for details).

An example where the function $G$ can be explicitly computed is a BRW with independent diffusion; in this case it is not difficult to see that $G(z \mid x)=\sum_{n \in \mathbb{N}} \rho_{x}(n)(P z(x))^{n}$, where $P z(x)=\sum_{y \in X} p(x, y) z(y)$. If, in particular,

$$
\rho_{x}(n)=\frac{1}{1+\bar{\rho}_{x}}\left(\frac{\bar{\rho}_{x}}{1+\bar{\rho}_{x}}\right)^{n}
$$

(as in the discrete-time counterpart of a continuous-time BRW) then the previous expression becomes $G(z \mid x)=\left(1+\bar{\rho}_{x} P(\mathbf{1}-z)(x)\right)^{-1}$. The previous equality can be written in a more 
compact way as

$$
G(z)=\frac{1}{1+M(1-z)},
$$

where $M$ is the first-moment matrix and $M v(x)=\bar{\rho}_{x} P \boldsymbol{v}(x)$ (by the definition of $P$ ). In (2.4) and hereafter, whenever $\boldsymbol{z}, \boldsymbol{v} \in[0,1]^{X}$, the ratio $\boldsymbol{z} / \boldsymbol{v}$ will be taken coordinatewise, that is, $(z / v)(x):=z(x) / v(x)$ for all $x$ such that $\boldsymbol{v}(x)>0$ (the value of $(z / v)(x)$ if $\boldsymbol{v}(x)=0$, if any, will be explicitly defined when needed).

When one is interested in whether a global surviving BRW survives strong locally, it may be useful to condition the process on global survival. Given a generic discrete-time BRW such that $\overline{\boldsymbol{q}}(x)<1$ for all $x \in X$, by conditioning on global survival we associate a BRW with no death (that is, a BRW such that $\rho_{x}(0)=0$ ). Let $\left\{\eta_{n}\right\}_{n \in \mathbb{N}}$ be the original BRW. Consider the event $\Omega_{\infty}=\left\{\sum_{x \in X} \eta_{n}(x)>0\right.$ for all $\left.n \in \mathbb{N}\right\}$, and define the process $\left\{\widehat{\eta}_{n}\right\}_{n \in \mathbb{N}}$ as follows: $\widehat{\eta}_{n}(x, \omega)$ equals the number of particles in $\eta_{n}(x, \omega)$ with at least one infinite line of descent when $\omega \in \Omega_{\infty}$, and it equals 0 when $\omega \notin \Omega_{\infty}$. Roughly speaking, $\left\{\widehat{\eta}_{n}\right\}_{n \in \mathbb{N}}$ is obtained from $\left\{\eta_{n}\right\}_{n \in \mathbb{N}}$ by removing all the particles with finite progeny, which are clearly irrelevant in view of the survival owing to the fact that $\overline{\boldsymbol{q}}(x)<1$ for all $x \in X$. Hence, the probability of local survival of $\left\{\widehat{\eta}_{n}\right\}_{n \in \mathbb{N}}$ in $A$ (for all $A \subseteq X$ ), starting from $x$, is equal to the same probability for $\left\{\eta_{n}\right\}_{n \in \mathbb{N}}$, that is, $\boldsymbol{q}(x, A)$. It can be shown that this process, restricted to $\Omega_{\infty}$, is a BRW that we call the no-death BRW associated to $\left\{\eta_{n}\right\}_{n \in \mathbb{N}}$ (we still denote it by $\left\{\widehat{\eta}_{n}\right\}_{n \in \mathbb{N}}$ ). Its generating function is

$$
\widehat{G}(z \mid x)=\frac{G(v(z) \mid x)-\overline{\boldsymbol{q}}(x)}{1-\overline{\boldsymbol{q}}(x)},
$$

where $G$ is the generating function of the original BRW and $v:[0,1]^{X} \rightarrow[0,1]^{X}$ is defined as $v(z \mid x):=\bar{q}(x)+\boldsymbol{z}(x)(1-\overline{\boldsymbol{q}}(x))$. In a more compact way, (2.5) can be written as $\widehat{G}=T_{\bar{q}}^{-1} \circ G \circ T_{\bar{q}}$, where $T_{\boldsymbol{w}}:[0,1]^{X} \rightarrow\left\{z \in[0,1]^{X}: \boldsymbol{w} \leq z\right\}$ is defined as $T_{\boldsymbol{w}} z(x):=$ $\boldsymbol{z}(x)(1-\boldsymbol{w}(x))+\boldsymbol{w}(x)$; note that $T_{\boldsymbol{w}}$ is nondecreasing and, if $\boldsymbol{w}(x)<1$ for all $x \in X$, bijective. In particular, if $\overline{\boldsymbol{q}}<\mathbf{1}$ then $T_{\overline{\boldsymbol{q}}}$ is a bijective map from the set of fixed points of $\widehat{G}$ to the set of fixed points of $G$.

Clearly, for all $A \subseteq X$, the probability of local survival in $A$ of the associated no-death BRW starting from $x$ is the probability of local survival in $A$ of the original BRW conditioned on global survival (starting from $x$ ), that is, $1-\left(T_{\overline{\boldsymbol{q}}}^{-1} \boldsymbol{q}(\cdot, A)\right)(x)=(1-\boldsymbol{q}(x, A)) /(1-\overline{\boldsymbol{q}}(x))$.

The following proposition is a sort of maximum principle for the function $(z-\bar{q}) /(\mathbf{1}-\overline{\boldsymbol{q}})$, where $z$ is such that $G(z) \geq \boldsymbol{z}$ (note that we are not assuming that $\overline{\boldsymbol{q}}(x)<1$ for all $x \in X$ ).

Proposition 2.1. Given $z \in[0,1]^{X}$ such that $z \geq \bar{q}$ is a solution of the inequality $G(z) \geq z$, we define $\widehat{\boldsymbol{z}}:=(z-\overline{\boldsymbol{q}}) /(\mathbf{1}-\overline{\boldsymbol{q}})$, where $\widehat{\boldsymbol{z}}(x):=1$ for all $x$ such that $\overline{\boldsymbol{q}}(x)=1$. Then, for all $x \in X$ such that the set $\mathcal{N}_{x}=\left\{y:(x, y) \in E_{\mu}\right\}$ is not empty, either $\widehat{z}(y)=\widehat{z}(x)$ for all $y \in \mathcal{N}_{x}$ or there exists $y \in \mathcal{N}_{x}$ such that $\widehat{\boldsymbol{z}}(y)>\widehat{\boldsymbol{z}}(x)$. In particular, if $\widehat{\boldsymbol{z}}(x)=1$ then, for all $y \in \mathcal{N}_{x}$, we have $\widehat{z}(y)=1$. The same results hold if we take the set $\{y \in X: x \rightarrow y\}$ instead of $\mathcal{N}_{x}$.

As an application, in a finite, final irreducible class (for instance, if the BRW is irreducible and the set $X$ is finite), if $z$ is as in Proposition 2.1 then $\widehat{z}$ is a constant vector.

\section{4. $\mathscr{F}$-BRWs}

Some results can be achieved if the BRW has some regularity; to this end, we introduce the concept of an $\mathcal{F}$-BRW (see also [28, Definition 4.2]), which extends the concept of quasitransitivity (see below). 
Definition 2.2. (a) A BRW $(X, \mu)$ is locally isomorphic to a $\mathrm{BRW}(Y, v)$ if there exists a surjective map $g: X \rightarrow Y$ such that $\nu_{g(x)}(\cdot)=\mu_{x}\left(\pi_{g}^{-1}(\cdot)\right)$, where $\pi_{g}: S_{X} \rightarrow S_{Y}$ is defined as $\pi_{g}(f)(y)=\sum_{z \in g^{-1}(y)} f(z)$ for all $f \in S_{X}$ and $y \in Y$.

(b) $(X, \mu)$ is an $\mathcal{F}$-BRW if it is locally isomorphic to some $\operatorname{BRW}(Y, v)$ on a finite set $Y$.

Clearly, if $(X, \mu)$ is locally isomorphic to $(Y, v)$ then

$$
G_{X}(z \circ g \mid x)=G_{Y}(z \mid g(x))
$$

for all $z \in[0,1]^{Y}$ and $x \in X$. We note that, since $\mu$ is uniquely determined by $G,(2.6)$ holds if and only if $(X, \mu)$ is locally isomorphic to $(Y, v)$ and $g$ is the map in Definition 2.2. If $\left\{\eta_{n}\right\}_{n \in \mathbb{N}}$ is a realization of the BRW $(X, \mu)$ then $\left\{\pi_{g}\left(\eta_{n}\right)\right\}_{n \in \mathbb{N}}$ is a realization of the $\operatorname{BRW}(Y, v)$.

Using (2.6) and the fact that $\overline{\boldsymbol{q}}=\lim _{n \rightarrow \infty} G^{(n)}(\mathbf{0})$ (see (3.1) with $A=X$ ), it is possible to prove that there is global survival for $(X, \mu)$ starting from $x$ if and only if there is global survival for $(Y, v)$ starting from $g(x)$ (see [28, Theorem 4.3]). It is not difficult to prove (the details can be found in [28] before Theorem 4.3) that, for all $x \in X, y \in Y$, and $n \in \mathbb{N}$, $\tilde{m}_{g(x) y}^{(n)}=\sum_{z \in g^{-1}(y)} m_{x z}^{(n)}$, where $\tilde{M}$ is the first-moment matrix of the $\operatorname{BRW}(Y, v)$. This implies that $M_{w}^{X}(x)=M_{w}^{Y}(g(x))$ for all $x \in X$.

In continuous time (see [8]) we can prove that $(X, K)$ is locally isomorphic to $(Y, \widetilde{K})$ if and only if there exists a surjective map $g: X \rightarrow Y$ such that $\sum_{z \in g^{-1}(y)} k_{x z}=\widetilde{k}_{g(x) y}$ for all $x \in X$ and $y \in Y$, whence $K_{w}^{X}(x)=K_{w}^{Y}(g(x))$ for all $x \in X$. In other words, the total rate at which particles at $x$ generate children and place them in the set of vertices with 'label' $y$, depends only on $y$ and on $g(x)$.

Roughly speaking, an $\mathcal{F}$-BRW is a BRW where the vertices of $X$ can be labeled by means of a finite alphabet $Y$ in such a way that the law of the labels of the positions of the children of a particle depends only on the label of the position of the parent. As an example, consider a graph $(X, E(X))$ such that $\sup _{x \in X} \operatorname{deg}(x)<+\infty$ and where $\operatorname{deg}(x)=\operatorname{deg}(y)$ implies that $\#\left\{z \in \mathcal{N}_{x}: \operatorname{deg}(z)=j\right\}=\#\left\{z \in \mathcal{N}_{y}: \operatorname{deg}(z)=j\right\}$ (for all $j$ ); an example of such a graph is a tree with two alternating degrees. In this case, a BRW on $X$ with independent diffusion where $\rho_{x}$ depends only on $\operatorname{deg}(x)$ is an $\mathcal{F}$-BRW and the label of $x$ is $\operatorname{deg}(x)$.

It is worth mentioning a particular subclass of $\mathcal{F}$-BRWs: a BRW is locally isomorphic to a branching process if and only if the law of the offspring number $\rho_{x}=\rho$ is independent of $x \in X$. In this case, the BRW is locally isomorphic to a BRW on a singleton $Y:=\{y\}$, where the law of the number of children of each particle is $\rho$ and $g(x):=y$ for all $x \in X$. The explicit computations of $M_{w}$ and $M_{s}$ in this case can be found after (2.1). In particular, a continuous-time BRW is locally isomorphic to a branching process if and only if $k(x)=k$ for all $x \in X$ (that is, if and only if it is a site-breeding BRW). In this case, $K_{w}(x)=k$ and $K_{S}(x, y)=k \lim \sup _{n \rightarrow \infty} \sqrt[n]{p^{(n)}(x, y)}$.

Let $\gamma: X \rightarrow X$ be an injective map. We say that $\mu=\left\{\mu_{x}\right\}_{x \in X}$ is $\gamma$-invariant if, for all $x \in X$ and $f \in S_{X}$, we have $\mu_{x}(f)=\mu_{\gamma(x)}\left(f \circ \gamma^{-1}\right.$ ) (where $f \circ \gamma^{-1}$ is extended to a function on $X$ by setting 0 outside $\gamma(X))$. In particular, a BRW with independent diffusion is $\gamma$-invariant if and only if $\rho_{x}=\rho_{\gamma(x)}$ and $p(x, y)=p(\gamma(x), \gamma(y))$ for all $x, y \in X$.

Moreover, $(X, \mu)$ is quasitransitive if and only if there exists a finite subset $X_{0} \subseteq X$ such that, for all $x \in X$, there exists a bijective map $\gamma: X \rightarrow X$ and $x_{0} \in X_{0}$ satisfying $\gamma\left(x_{0}\right)=x$, and $\mu$ is $\gamma$-invariant. An edge-breeding BRW on a graph $(X, E)$ is quasitransitive if and only if $(X, E)$ is a quasitransitive graph.

We note that every quasitransitive BRW is an $\mathcal{F}$-BRW (see [28, Section 6.2]). The class of $\mathcal{F}$-BRWs is strictly larger than the class of quasitransitive BRWs. For instance, consider the 


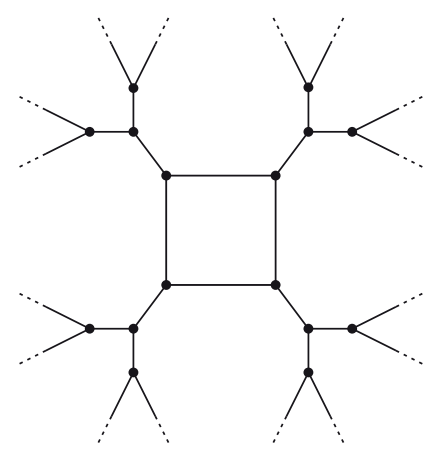

(a)

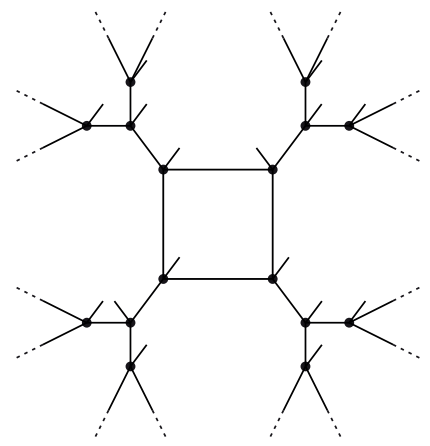

(b)

FiguRE 1: (a) A regular graph of degree 3. (b) An irregular graph.

BRW described in Example 4.4. Indeed, in this case the BRW is $\gamma$-invariant if and only if, for all $i, j \in \mathbb{N}, p(\gamma(i), \gamma(j))=p(i, j)$. This implies that $\gamma(0)=0$ and, by induction, $\gamma(i)=i$ for all $i \in \mathbb{N}$. Thus, the only invariant map $\gamma$ is the identity on $\mathbb{N}$, whence there is no finite $X_{0}$ as described in the definition of quasitransitivity. Nevertheless, the BRW is locally isomorphic to a branching process; thus, it is an $\mathcal{F}$-BRW. Other examples are the edge-breeding BRWs associated to the following graphs: take a square and attach to each vertex an infinite branch of a homogeneous tree $\mathbb{T}_{3}$ of degree 3 (see Figure 1(a)); now attach to each vertex of the new graph an edge with a new endpoint (see Figure 1(b)). They are both $\mathcal{F}$-BRWs which are not quasitransitive; moreover, while the first graph is regular (it has constant degree 3 ), the second graph is not, since it has vertices with degree 4 and vertices with degree 1.

\section{Conditions for survival and extinction}

\subsection{Local and global survival}

The following theorems summarize the main results about local and global survival for discrete-time BRWs and continuous-time BRWs respectively (see [8, Theorems 4.1, 4.7, and 4.8, and Proposition 4.5] and [28, Theorems 4.1 and 4.3]). In particular, Theorem 3.1(d) is a straightforward generalization of [6, Theorem 3.6] (we omit the proof).

Theorem 3.1. Let $(X, \mu)$ be a discrete-time $B R W$.

(a) There is local survival starting from $x$ if and only if $M_{S}(x, x)>1$.

(b) There is global survival starting from $x$ if and only if there exists $z \in[0,1]^{X}, z(x)<1$ such that $G(z \mid y)=z(y)$ for all $y \in X$ (equivalently, such that $G(z \mid y) \leq z(y)$ for all $y \in X)$.

(c) If $(X, \mu)$ is an $\mathcal{F}-B R W$, there is global survival starting from $x$ if and only if $M_{w}(x)>1$.

(d) If $(X, \mu)$ is an irreducible, nonoriented $\mathcal{F}-B R W$ then $M_{s}<M_{w}$ if and only if $(X, \mu)$ is nonamenable.

Note that whether or not there is local survival depends only on the first-moment matrix $M$. In particular, the BRW survives locally at $x$ if and only if it does so when restricted to the irreducibility class $[x]$ of $x$. It is worth noting that if $[x]$ is finite then $M_{s}(x, x)$ is the PerronFrobenius eigenvalue of the submatrix $M^{\prime}:=\left(m_{y z}\right)_{y, z \in[x]}$. In this case there is local survival 
at $x$ if and only if $\max \left\{t>0\right.$ : there exists $\left.v \neq \mathbf{0}, M^{\prime} v=t v\right\}>1$. In general, the global behavior does not depend only on $M$ (see [28, Example 4.4]) unless there is a one-to-one correspondence between first moment matrices and processes. This is true for instance in the class of BRWs with independent diffusion such that

$$
\rho_{x}(n)=\frac{1}{1+\bar{\rho}_{x}}\left(\frac{\bar{\rho}_{x}}{1+\bar{\rho}_{x}}\right)^{n}
$$

(hence for a continuous-time BRW). Indeed, in that case an equivalent condition for global survival starting from $x \in X$ is the existence of $v \in[0,1]^{X}, \boldsymbol{v}(x)>0$ such that

$$
M v \geq \frac{v}{1-v} \quad\left(\text { equivalently, } M v=\frac{v}{1-v}\right),
$$

which comes from Theorem 3.1(b) given $z:=\mathbf{1}-\boldsymbol{v}$ and the explicit expression (2.4) of $G$. In particular, for a BRW with independent diffusion, the local survival probability $\boldsymbol{v}_{A}:=$ $\mathbf{1}-\boldsymbol{q}(\cdot, A)$ satisfies $M \boldsymbol{v}_{A}=\boldsymbol{v}_{A} /\left(\mathbf{1}-\boldsymbol{v}_{A}\right)$, which becomes $\lambda K \boldsymbol{v}_{A}=\boldsymbol{v}_{A} /\left(\mathbf{1}-\boldsymbol{v}_{A}\right)$ for a continuous-time BRW.

Theorem 3.2. Let $(X, K)$ be a continuous-time BRW.

(a) $\lambda_{s}(x)=1 / K_{S}(x, x)$ and if $\lambda=\lambda_{s}(x)$ then there is local extinction at $x$.

(b) $\lambda_{w}(x) \geq 1 / K_{w}(x)$.

(c) If $(X, K)$ is an $\mathcal{F}$-BRWs then $\lambda_{w}(x)=1 / K_{w}(x)$, and when $\lambda=\lambda_{w}(x)$, there is global extinction starting from $x$.

(d) If $(X, K)$ is an irreducible, nonoriented $\mathcal{F}-B R W$ then $\lambda_{s}>\lambda_{w}$ if and only if $(X, \mu)$ is nonamenable.

For a generic BRW when $\lambda=\lambda_{w}(x)$, there might be global survival (see [8, Example 3]). A characterization of $\lambda_{w}(x)$ has been given in [8, Theorem 4.2] by means of the so-called lower Collatz-Wielandt number.

\subsection{Probabilities of extinction and strong local survival}

Define $\boldsymbol{q}_{n}(x, A)$ as the probability of extinction in $A$ no later than the $n$th generation, starting with one particle at $x$, namely, $\boldsymbol{q}_{n}(x, A)=\mathbb{P}^{\delta_{x}}\left(\eta_{k}(y)=0\right.$ for all $k \geq n$ and all $\left.y \in A\right)$. It is clear that $\left\{\boldsymbol{q}_{n}(x, A)\right\}_{n \in \mathbb{N}}$ is a nondecreasing sequence satisfying

$$
\boldsymbol{q}_{n}(\cdot, A)=G\left(\boldsymbol{q}_{n-1}(\cdot, A)\right) \quad \text { for all } n \geq 1, \quad \boldsymbol{q}_{0}(x, A)=0 \quad \text { for all } x \in A ;
$$

hence, there is a limit $\boldsymbol{q}(x, A)=\lim _{n \rightarrow \infty} \boldsymbol{q}_{n}(x, A) \in[0,1]^{X}$ which is the probability of local extinction in $A$, starting with one particle at $x$ (see Definition 2.1). Note that (3.1) completely defines the sequence $\left\{\boldsymbol{q}_{n}(\cdot, A)\right\}_{n \in \mathbb{N}}$ only when $A=X$ (otherwise, we need the values $\boldsymbol{q}_{0}(x, A)$ for $x \notin A)$. Since $G$ is continuous, we have $\boldsymbol{q}(\cdot, A)=G(\boldsymbol{q}(\cdot, A))$; hence, these extinction probabilities are fixed points of $G$.

Note that $\boldsymbol{q}(\cdot, \varnothing)=\mathbf{1}$. Since $\overline{\boldsymbol{q}}=\lim _{n \rightarrow \infty} G^{(n)}(\mathbf{0}), \overline{\boldsymbol{q}}$ is the smallest fixed point of $G$ in $[0,1]^{X}$ (see [8, Corollary 2.2]). Using the same arguments, we can prove that $\overline{\boldsymbol{q}}$ is the smallest fixed point of $G^{(m)}$ for all $m \in \mathbb{N}$.

Note that $A \subseteq B \subseteq X$ implies that $\boldsymbol{q}(\cdot, A) \geq \boldsymbol{q}(\cdot, B) \geq \overline{\boldsymbol{q}}$. Since, for all finite $A \subseteq X$, we have $\boldsymbol{q}(x, A) \geq 1-\sum_{y \in A}(1-\boldsymbol{q}(x, y))$, then, for any given finite $A \subseteq X, \boldsymbol{q}(x, A)=1$ if and only if $\boldsymbol{q}(x, y)=1$ for all $y \in A$. 
If $x \rightarrow x^{\prime}$ and $A \subseteq X$ then $\boldsymbol{q}\left(x^{\prime}, A\right)<1$ implies that $\boldsymbol{q}(x, A)<1$; as a consequence, if $x \rightleftharpoons x^{\prime}$ then $\boldsymbol{q}(x, A)<1$ if and only if $\boldsymbol{q}\left(x^{\prime}, A\right)<1$. Moreover, if $y \rightleftharpoons y^{\prime}$, we have $\boldsymbol{q}(x, y)=\boldsymbol{q}\left(x, y^{\prime}\right)$ for all $x \in X$.

In the irreducible case, if $\rho_{x}(0)>0$ for all $x \in X$, we have $\overline{\boldsymbol{q}}(x)=\boldsymbol{q}(x, A)$ for some $x \in X$ and a finite subset $A \subseteq X$ if and only if $\overline{\boldsymbol{q}}(y)=\boldsymbol{q}(y, B)$ for all $y \in X$ and all finite subsets $B \subseteq X$ (hence, strong local survival is a common property of all subsets and all starting vertices). Clearly, this may not be true in the reducible case. Besides, if we drop the assumption that $\rho_{x}(0)>0$ for all $x \in X$, we might actually have $\overline{\boldsymbol{q}}(x)=\boldsymbol{q}(x, A)<1$ and $\overline{\boldsymbol{q}}(y)<\boldsymbol{q}(y, A)$ for some $x, y \in X$ and a finite $A \subseteq X$ even when the BRW is irreducible (see Example 4.3). Hence, in general, even for irreducible BRWs, strong local survival is not a common property of all vertices, as local and global survival are.

The following theorem, in the case of global survival, gives equivalent conditions for strong local survival in terms of extinction probabilities.

Theorem 3.3. The following assertions are equivalent for every nonempty subset $A \subseteq X$.

(a) $\boldsymbol{q}(x, A)=\overline{\boldsymbol{q}}(x)$ for all $x \in X$.

(b) $\boldsymbol{q}_{0}(x, A) \leq \overline{\boldsymbol{q}}(x)$ for all $x \in X$.

(c) The probability of visiting A at least once starting from $x$ is greater than or equal to the probability of global survival starting from $x$ for all $x \in X$.

(d) For all $x \in X$, either $\overline{\boldsymbol{q}}(x)=1$ or the probability of visiting A at least once starting from $x$ conditioned on global survival starting from $x$ is 1 .

(e) For all $x \in X$, either $\overline{\boldsymbol{q}}(x)=1$ or the probability of local survival in A starting from $x$ conditioned on global survival starting from $x$ is 1 (strong local survival in A starting from $x$ ).

From this theorem, it follows that if there exists $x \in X$ such that $\boldsymbol{q}(x, A)>\overline{\boldsymbol{q}}(x)$ (that is, there is a positive probability of global survival and nonlocal survival in $A$ starting from $x$ ) then there exists $y \in X$ such that $\boldsymbol{q}_{0}(y, A)>\overline{\boldsymbol{q}}(y)$ (that is, there is a positive probability that the colony survives globally starting from $y$ without ever visiting $A)$. Note that $\boldsymbol{q}_{0}(x, A)>\overline{\boldsymbol{q}}(x)$ implies that $\boldsymbol{q}(x, A)>\overline{\boldsymbol{q}}(x)$, but the converse is not true. In particular, for a BRW with no death, there is strong local survival in $A$ starting from $x$ for all $x \in X$ if and only if the probability of visiting $A$ is 1 starting from every vertex.

We note that, a priori, there is no order relation between the events 'visiting $A$ at least once starting from $x$ ' and 'global survival starting from $x$ '. Nevertheless, if, for all $x \in X$, the probability of 'visiting $A$ at least once starting from $x$ ' is greater than or equal to the probability of 'global survival starting from $x$ ', then, by Theorem 3.3, the probability of 'global survival starting from $x$ never visiting $A$ ' is 0 and this implies, whenever $\overline{\boldsymbol{q}}(x)<1$, that there is strong local survival in $A$ starting from $x$.

In the case of an $\mathcal{F}$-BRW, the fixed points of $G$ have an interesting property, stated in the following theorem.

Theorem 3.4. Let $(X, \mu)$ be an $\mathcal{F}-B R W$.

(a) There exists at most one fixed point $z$ for $G$ such that $\sup _{x \in X} z(x)<1$, namely, $z=\bar{q}$.

(b) For all $x \in X$, either $\boldsymbol{q}(\cdot, x)=\overline{\boldsymbol{q}}(\cdot)$ or $\sup _{w \in X} \boldsymbol{q}(w, x)=1$. In particular, when $(X, \mu)$ is irreducible then either $\boldsymbol{q}(x, x)=\overline{\boldsymbol{q}}(x)$ for all $x \in X$ or $\sup _{x \in X} \boldsymbol{q}(x, x)=1$. 
It is worth noting that, unlike the branching process, for a generic irreducible $\mathcal{F}$-BRW, when $\overline{\boldsymbol{q}}<\mathbf{1}$, there might be other fixed points for $G$ (see Examples 4.2, 4.4, and 4.5, and Remark 4.1). Nevertheless, this cannot happen when $X$ is finite.

Corollary 3.1. If $X$ is finite and the BRW is irreducible, then there are at most two solutions of $G(z) \geq \boldsymbol{z}$ when $z \geq \overline{\boldsymbol{q}}$, that is, $\overline{\boldsymbol{q}}$ and $\mathbf{1}$.

Using Theorem 3.4, we can describe the case when $X$ is finite (not necessarily irreducible). Clearly, in this case $\overline{\boldsymbol{q}}(w)=\boldsymbol{q}\left(w, A_{w}\right)$, where $A_{w}:=\{x \in X: w \rightarrow x\}$. Moreover, for all $x \in X$, either $\boldsymbol{q}(\cdot, x)=\overline{\boldsymbol{q}}(\cdot)$ or there exists $w \in X$ such that $\boldsymbol{q}(w, x)=1$. If the BRW is irreducible (and $X$ is finite) then $\overline{\boldsymbol{q}}(w)=\boldsymbol{q}(w, w)$ for all $w \in X$ or $\boldsymbol{q}(w, x)=1$ for all $w, x \in X$.

Corollary 3.2. Let $(X, \mu)$ be an irreducible and quasitransitive BRW. Then the existence of $x \in X$ such that there is local survival at $x($ i.e. $q(x, x)<1)$ implies that there is strong local survival at $y$ starting from $w$ for every $w, y \in X($ i.e. $\boldsymbol{q}(w, y)=\overline{\boldsymbol{q}}(w))$.

Hence, for a quasitransitive, irreducible BRW, whenever there is local survival, it is a strong local survival; in continuous time this implies that there is global and local extinction if $\lambda \in$ $\left[0, \lambda_{w}\right]$, pure global survival if $\lambda \in\left(\lambda_{w}, \lambda_{s}\right]$, and strong local survival if $\lambda \in\left(\lambda_{s},+\infty\right)$ (see also Theorem 3.2).

In the particular case of a quasitransitive, irreducible BRW with no death and with independent diffusion, Corollary 3.2 was proved in [23, Theorem 3.7]. The proof we give in Section 5 is of a different nature. Unlike Theorem 3.4, Corollary 3.2 does not hold for every $\mathcal{F}$-BRW; indeed, as Examples 4.4 and 4.5 below show, for an irreducible $\mathcal{F}$-BRW, there might be nonstrong local survival.

The following result follows by applying [21, Theorem 3.1] to the no-death BRW associated to a generic BRW as described in Section 2.3 (hence we omit the proof). The original result [21, Theorem 3.1] can be recovered from this result by assuming that $\rho_{x}(0)=0$ for all $x \in X$, which implies that $\overline{\boldsymbol{q}}=\mathbf{0}$ and $T_{\overline{\boldsymbol{q}}}^{-1}$ is equal to the identity.

Theorem 3.5. Let $(X, \mu)$ be an irreducible, globally surviving BRW. Then there is no strong local survival if and only if there exists a finite, nonempty set $A \subseteq X$ and a function $v \in[0,1]^{X}$ such that $\overline{\boldsymbol{q}} \leq \boldsymbol{v}$ and

$$
G(\boldsymbol{v} \mid x) \geq \boldsymbol{v}(x) \text { for all } x \in A^{\complement}, \quad\left(T_{\overline{\boldsymbol{q}}}^{-1} \boldsymbol{v}\right)\left(x_{0}\right)>\max _{x \in A}\left(T_{\overline{\boldsymbol{q}}}^{-1} \boldsymbol{v}\right)(x) \text { for some } x_{0} \in A^{\complement},
$$

where $T_{\overline{\boldsymbol{q}}}^{-1} \boldsymbol{v}=(\boldsymbol{v}-\overline{\boldsymbol{q}}) /(\mathbf{1}-\overline{\boldsymbol{q}})$.

\subsection{Pure global survival}

The idea of pure global survival (see Definition 2.1(d)) was first introduced in continuoustime BRW theory (and, more generally, in interacting particle theory) to define the situation where $\lambda_{s}(x)>\lambda_{w}(x)$. In this case, for every $\lambda \in\left(\lambda_{w}(x), \lambda_{s}(x)\right]$, there is a positive probability of global survival starting from $x$, but the colony dies out locally at $x$ almost surely. A necessary condition for the existence of a pure global survival phase starting from $x$ is $K_{s}(x, x)<$ $K_{w}(x)$ (see Theorem 3.2). According to Theorem 3.2(c), for an $\mathcal{F}$-BRW, this condition is also sufficient.

Clearly, for an irreducible, continuous-time BRW, the existence of pure global survival does not depend on the starting vertex since $\lambda_{w}(x)=\lambda_{w}$ and $\lambda_{s}(x)=\lambda_{s}$ for all $x \in X$. This is still true for an irreducible discrete-time BRW as a consequence of Proposition 2.1. Indeed, if 
$\boldsymbol{z}(\cdot):=\boldsymbol{q}(\cdot, A), \widehat{\boldsymbol{z}}(x)$ can be interpreted as the probability of local extinction in $A$ conditioned on global survival (starting from $x$ ). Thus, according to Proposition 2.1, if the BRW is irreducible then this conditional probability is 1 everywhere, provided it is 1 somewhere. This means that if there is pure global survival starting from some $x$ then there is pure global survival starting from every $x$.

Theorem 3.2(d) tells us that an irreducible, continuous-time $\mathcal{F}$-BRW has a pure global survival phase if and only if it is nonamenable. This is not true if the process is not an $\mathcal{F}$-BRW, as shown by Example 4.1. The same example shows that pure global survival is a fragile property of a BRW. Indeed, finite modifications, such as, for an edge-breeding BRW, attaching a complete finite graph to a vertex or removing a set of vertices and/or edges can create it or destroy it.

\section{Examples}

The first example shows that there are irreducible amenable BRWs with pure global survival and irreducible nonamenable BRWs with no pure global survival (see also [24]).

Example 4.1. In this example we frequently use the following argument (which is an adaptation from [6, Remark 3.2]). Consider a continuous-time BRW adapted to a connected graph $X$, in the sense that $k_{x y}>0$ if and only if $(x, y)$ is an edge. In some cases, it is easy to show that the existence of a pure global survival of a BRW implies the existence of a pure global survival of the BRW restricted to some subgraph (where all the rates $k_{x y}$ are turned to 0 if $x$ or $y$ do not belong to the subgraph). Indeed, if $Y$ is a finite subset of $X$ such that $X \backslash Y$ is divided into a finite number of connected graphs $X_{1}, \ldots, X_{n}$ (which is certainly true if $k_{x y}>0$ is equivalent to $k_{y x}>0$ for all $\left.x, y \in X \backslash Y\right)$ then, for every $\lambda \in\left(\lambda_{w}^{X}, \lambda_{s}^{X}\right)$, the BRW on $X$ eventually leaves, almost surely, the subset $Y$. Hence, it survives (globally but not locally) at least on one connected component; this means that, although $\lambda_{s}^{X_{i}} \geq \lambda_{s}^{X}$ and $\lambda_{w}^{X_{i}} \geq \lambda_{w}^{X}$ for all $i=1, \ldots, n$ (since $X_{i} \subseteq X$ ), there exists $i_{0}$ such that $\lambda_{w}^{X_{i_{0}}}=\lambda_{w}^{X}$. The existence of a pure global survival on $X_{i_{0}}$ follows from $\lambda_{s}^{X_{i_{0}}} \geq \lambda_{s}^{X}>\lambda_{w}^{X}=\lambda_{w}^{X_{i_{0}}}$. Hence, if there exists a subset $Y$ as above such that $\lambda_{w}^{X_{i}}>\lambda_{w}^{X}$ for all $i$ then there is no pure global survival for the BRW on $X$.

Consider an irreducible, edge-breeding continuous-time BRW on the (nonoriented) graph $X$ obtained by attaching to a copy of $\mathbb{N}$ one branch $T$ of the homogeneous tree $\mathbb{T}_{3}$. The BRW is amenable by the presence of the copy of $\mathbb{N}$. We claim that $\lambda_{s}^{X}=\lambda_{s}^{\mathbb{T}_{3}}$ and $\lambda_{w}^{X}=\lambda_{w}^{\mathbb{T}_{3}}$. Indeed, $T \subset X \subset \mathbb{T}_{3}$; hence, $\lambda_{s}^{T} \geq \lambda_{s}^{X} \geq \lambda_{s}^{\mathbb{T}_{3}}$ and $\lambda_{w}^{T} \geq \lambda_{w}^{X} \geq \lambda_{w}^{\mathbb{T}_{3}}$. But, by approximation, $\lambda_{s}^{T}=\lambda_{s}^{\mathbb{T}_{3}}$. Indeed, $\lambda_{s}^{T} \geq \lambda_{s}^{\mathbb{T}_{3}}$ and it does not depend on the starting vertex. Moreover, $T$ contains arbitrarily large balls isomorphic to balls of $\mathbb{T}_{3}$; hence, by [28, Theorem 5.2] or [7, Theorem 3.1], their critical local parameters coincide. Note that, in [28, Section 5.1], the hypothesis that $M$ is a nonnegative matrix is not stated, even though it is implicitly used. Moreover, [28, Theorems 5.1 and 5.2] hold without the irreducibility hypothesis. The key is that, given a sequence $\left\{X_{n}\right\}_{n \in \mathbb{N}}$ of subsets of $X$ such that $\liminf _{n} X_{n}=X$, and defining the sequence of matrices $\left.{ }_{n} M\right\}_{n \in \mathbb{N}}$ as ${ }_{n} M:=\left(m_{x, y}\right)_{x, y \in X_{n}}$, we can prove that, for all $x_{0} \in X,\left({ }_{n} M\right)_{s}\left(x_{0}, x_{0}\right) \rightarrow M_{s}\left(x_{0}, x_{0}\right)$ (as defined in (2.1)). Explicit computations show that $\lambda_{w}^{\mathbb{T}_{3}}=\frac{1}{3}<1 / 2 \sqrt{2}=\lambda_{s}^{\mathbb{T}_{3}}$ (there is pure global survival on $\mathbb{T}_{3}$ ). Since $\mathbb{T}_{3}$ can be obtained by attaching three copies of $T$ to a root, the above discussion about surviving on a subgraph implies that $\lambda_{w}^{T}=\lambda_{w}^{\mathbb{T}_{3}}$. Then we have $\lambda_{w}^{X}=\lambda_{w}^{T} \leq \lambda_{w}^{\mathbb{T}_{3}}<\lambda_{s}^{\mathbb{T}_{3}}=\lambda_{s}^{X}$, and there is pure global survival on $X$.

On the other hand, consider a nonamenable graph $X^{\prime}$ such that the corresponding edgebreeding continuous-time BRW has a pure global survival phase (take, for instance, $X^{\prime}:=\mathbb{T}_{3}$, 
the homogeneous tree with degree 3). Attach to a vertex of $X^{\prime}$ a complete graph $Y$ with degree $k>1 / \lambda_{w}^{X^{\prime}}$ by an edge. It is easy to show that the resulting graph $X$ is still nonamenable; nevertheless, there is no pure global survival for the corresponding edge-breeding BRW. Indeed, by the above discussion, if there were pure global survival on $X$ then one of the connected components of $X \backslash Y$ should have the same global critical value of $X$; but $X \backslash Y=X^{\prime}$ and $\lambda_{w}^{X} \leq 1 / k<\lambda_{w}^{X^{\prime}}$. Roughly speaking, for every $\lambda \in\left(\lambda_{w}^{X}, \lambda_{w}^{X^{\prime}}\right)$, the process cannot survive globally in $X^{\prime}$; hence, with positive probability, it infinitely often hits the complete graph, and, thus, $\lambda_{s}^{X}=\lambda_{w}^{X}$.

The following example shows that the strong local survival is not monotone. The counterexample is obtained by modifying the edge-breeding BRW on a particular graph, namely the homogeneous tree $\mathbb{T}_{d}$. The crucial property that we need here is the existence of a pure global survival phase; thus, the procedure applies to every BRW with such a phase.

Example 4.2. Consider the edge-breeding continuous-time BRW on the homogeneous tree $\mathbb{T}_{d}$ with degree $d \geq 3$. Since the graph has constant degree $d$, the BRW can be seen also as a site-breeding process where $k(x)=d$ for all $x \in \mathbb{T}_{d}$. Hence, it is locally isomorphic to a branching process, which implies that $\lambda_{w}(x)=1 / K_{w}(x)=1 / d$ for all $x \in \mathbb{T}_{d}$, and if $\lambda \leq 1 / d$ then the probabilities of survival are 0 (see Theorem 3.2(c)). Similarly, according to Theorem 3.2(a), $\lambda_{s}(x)=1 / K_{S}(x, x)$, which does not depend on $x$. By the definition of $P$ and the discussion after $(2.3), K_{S}(x, x)=d \lim _{\sup _{n \rightarrow \infty}} \sqrt[n]{p^{(n)}(x, y)}$, where $P$ is the diffusion matrix of the simple random walk on $\mathbb{T}_{d}$. Using [27, Lemma 1.24], we obtain $K_{s}(x, x)=2 \sqrt{d-1}$, which implies that $\lambda_{s}(x)=1 / 2 \sqrt{d-1}$ for all $x \in \mathbb{T}_{d}$ (and there is global extinction when $\lambda=\lambda_{w}$ ). Hence, if $\lambda>1 / 2 \sqrt{d-1}$, there is strong local survival (see Corollary 3.2), while if $\lambda \in(1 / d, 1 / 2 \sqrt{d-1}]$, the probability of global survival is positive and independent of the starting point, and the probability of local survival at any finite $A \subseteq X$ is 0 .

Fix $\lambda \in(1 / d, 1 / 2 \sqrt{d-1}]$ and a finite $A \subseteq X$. According to Theorem 3.3, there exists $x \in X$ such that there is a positive probability of global survival starting from $x$ without ever visiting $A$ (clearly, $x \notin A$ ). In this case, any modification of the rates in the subset $A$ provides a new BRW such that there is still a positive probability of global survival starting from $x$ without ever visiting $A$ (since the original BRW and the new one coincide until the first hitting time on $A$ ). On the other hand, if there is $y \in A$ such that $x \rightarrow y$, and we add a loop in $y$ and a rate $k_{y y}>1 / \lambda$, then $\overline{\boldsymbol{q}}(x)<\boldsymbol{q}(x, y)<1$. The first inequality holds by the above discussion on local modifications, and the second inequality holds since $\lambda k_{y y}>1$ implies local survival at $y$ (then irreducibility implies local survival at $y$ starting from $x$ ). This means that, for this fixed value of $\lambda$, we obtained a locally and globally (but not strong-locally) surviving BRW at $y$ starting from $x$.

Suppose now that $k_{y y}>d$. Then, as in Example 4.1, we have a new BRW such that $\lambda_{w}^{\prime}=$ $\lambda_{s}^{\prime} \leq 1 / k_{y y}$. In this case, when $\lambda \leq \lambda_{w}^{\prime}$, there is global extinction. When $\lambda>1 / 2 \sqrt{d-1}$, there is strong local survival for the new BRW since there is strong local survival for the original BRW (the probability of hitting $x$ conditioned on global survival is 1 for both processes and Theorem 3.3 applies). If $\lambda \in\left(\lambda_{w}^{\prime}, 1 / d\right]$, there is local and global survival with the same probability, since, in order to survive globally, the process must visit $x$ infinitely many times (it cannot survive globally in the branches of $\mathbb{T}_{d}$; otherwise, according to Theorem 3.3 , both processes would survive globally w.p.p. without ever hitting $x)$. If $\lambda \in(1 / d, 1 / 2 \sqrt{d-1}]$ then $k_{y y}>1 / \lambda$ and, according to the previous discussion, there is nonstrong local survival for the new BRW. 
We show that, even in the irreducible case, if $\rho_{x}(0)=0$ for some $x \in X$ then we might have strong local survival starting from some vertices and not from others.

Example 4.3. Let us consider a modification of the discrete-time counterpart of the edgebreeding BRW on $\mathbb{T}_{d}$ with degree $d \geq 3$ and $\lambda \in(1 / d, 1 / 2 \sqrt{d-1}]$. Let us fix a vertex $y$; in this modified version we add, with probability 1 , one child at $y$ for every particle at $y$. In this case, $\overline{\boldsymbol{q}}(y)=\boldsymbol{q}(y, A)=0$ for all $A \subseteq X$. On the other hand, as in Example 4.2, there is a vertex $x$ such that $\overline{\boldsymbol{q}}(x)<\boldsymbol{q}(x, y)$.

In the last few examples we make use of the subclass of BRWs which are locally isomorphic to a branching process (which are particular $\mathcal{F}$-BRWs; see Section 2.4). By using Theorems 3.1 and 3.2, and the explicit computations for $M_{s}$ and $M_{w}$ given after (2.1), it is easy to show that, for such a process, (i) there is global survival if and only if $\bar{\rho}>1$; (ii) there is local survival at $x$ if and only if $\bar{\rho}>1 / \lim \sup _{n \rightarrow \infty} \sqrt[n]{p^{(n)}(x, x)}=: r(x, x)$. Hence, in the irreducible case, there is pure global survival if and only if $1<\bar{\rho} \leq r$ (where $r=r(x, x)$ in this case does not depend on $x \in X$ due to irreducibility). This is possible if and only if $r>1$, which is equivalent to nonamenability, since in this case $M_{S}(x, y)=\bar{\rho} / r$ and $M_{w}(x)=\bar{\rho}$. It is clear that, given a continuous-time BRW which is locally isomorphic to a branching process, $\lambda_{w}=1 / \mathrm{k}$ and $\lambda_{s}(x)=r(x, x) / k($ where $k=k(x)$ for all $x \in X)$.

In general, there may be nonstrong local survival, even if the BRW is irreducible and locally isomorphic to a branching process, and has independent diffusion as Examples 4.4 and 4.5 show (see also Example 4.2). This disproves [25, Theorem 3 and Corollary 4] (see also Remark 4.1) since $\overline{\boldsymbol{q}}<\boldsymbol{q}(\cdot, 0)<\mathbf{1}$ are three distinct fixed points of $G$.

Example 4.4. Fix $X:=\mathbb{N}$, and consider a BRW with the following reproduction probabilities. Every particle has two children with probability $\frac{3}{4}$ and no children with probability $\frac{1}{4}$. Each newborn particle is dispersed independently according to a nearest-neighbor matrix $P$ on $\mathbb{N}$. More precisely,

$$
p(i, j):= \begin{cases}p_{i} & \text { if } j=i+1, \\ 1-p_{i} & \text { if } j=i-1,\end{cases}
$$

and $p_{0}=1$. The process described above is an irreducible $\mathcal{F}$-BRW for every choice of the set $\left\{p_{i}\right\}_{i \in \mathbb{N} \backslash\{0\}}$ such that $p_{i} \in(0,1)$ for all $i>0$. The generating function of the total number of children is $z \mapsto 3 z^{2} / 4+\frac{1}{4}$ and its minimal fixed point is $\frac{1}{3}=\overline{\boldsymbol{q}}(x)$ (for all $x \in \mathbb{N}$ ).

Choose $p_{1}<\frac{5}{9}$. It is easy to show that the process confined to $\{0,1\}$ (that is, every particle sent outside $\{0,1\}$ is killed) survives, since the expected number of children at 0 every two generations (starting from 0 ) is $\left(\frac{3}{2}\right)^{2}\left(1-p_{1}\right)>1$. Since the confined process is stochastically dominated by the original process, we have local survival, for instance, at $x=0$. By irreducibility, this implies that $\boldsymbol{q}(x, y)<1$ and $\overline{\boldsymbol{q}}(x)<1$ for all $x, y \in \mathbb{N}$.

Choose the $p_{i}$ such that $\prod_{i=1}^{\infty} p_{i}^{2^{i}}>0$ (or, equivalently, $\left.\sum_{i=1}^{\infty} 2^{i}\left(1-p_{i}\right)<+\infty\right)$. Consider the branching process $N_{n}$ representing the total number of particles alive at time $n$ : for all $n$, $N_{n} \leq 2^{n}$ almost surely. The probability, conditioned on global survival, that every particle places its children (if any) to its right is the conditional expected value of $\prod_{i=1}^{\infty} p_{i}^{N_{i}}$. But $\prod_{i=1}^{\infty} p_{i}^{N_{i}} \geq \prod_{i=1}^{\infty} p_{i}^{2^{i}}>0$ almost surely. Hence, conditioning on global survival, there is a positive probability of nonlocal survival. This implies that $\boldsymbol{q}(\cdot, y) \neq \overline{\boldsymbol{q}}$ for every $y \in \mathbb{N}$. Note that, according to Theorem 3.4, $\sup _{x \in \mathbb{N}} \boldsymbol{q}(x, x)=1$. This proves that, even in the irreducible case, the generating function $G$ can have more than two fixed points (see also Remark 4.1). 
The key in the previous example is that the total number of particles alive at time $n$ is bounded. This is not an essential assumption. The following example shows that, given any law $\rho$ of a surviving branching process (that is, $\bar{\rho}=\sum_{n \in \mathbb{N}} n \rho(n)>1$ ), it is possible to construct an irreducible BRW which is locally isomorphic to a branching process with nonstrong local survival.

Example 4.5. Let $X=\mathbb{N}$ and $\rho_{x}:=\rho$ for all $x \in \mathbb{N} ; \rho$ being the law of a surviving branching process. We know that $\overline{\boldsymbol{q}}(x) \equiv \bar{q}$ for all $x \in \mathbb{N}$, where $\bar{q}<1$ is the smallest fixed point of $z \mapsto \sum_{n \in \mathbb{N}} \rho(n) z^{n}$. Pick a sequence of natural numbers $\left\{N_{i}\right\}_{i \in \mathbb{N}}$ satisfying

$$
\prod_{i \in \mathbb{N}} \rho\left(\left[0, N_{i+1}\right]\right)^{\prod_{j=0}^{i} N_{j}}>\bar{q},
$$

where $N_{0}:=1$. Note that the probability of the event $\mathcal{A}=$ 'every particle alive at time $i$ has at most $N_{i+1}$ children for all $i \in \mathbb{N}^{\prime}$ is bounded from below by the left-hand side of (4.1). Thus, from (4.1), with a probability greater than $\prod_{i \in \mathbb{N}} \rho\left(\left[0, N_{i+1}\right]\right) \prod_{j=0}^{i} N_{j}-\bar{q}>0$, the colony survives globally and the total size of the population at time $n$ is not greater than $\prod_{j=0}^{n} N_{j}$ (i.e. the intersection between $\mathcal{A}$ and global survival has positive probability).

We define a BRW with independent diffusion, with $P$ defined as

$$
p(i, j):= \begin{cases}p_{i}, & j=i+1, i \geq 0 \\ 1-p_{i}, & j=i-1, i \geq 1 \\ 1-p_{0}, & i=j=0\end{cases}
$$

Let $p_{0}$ such that $\left(1-p_{0}\right) \bar{\rho}>1$; this implies local survival. We choose the sequence $\left\{p_{i}\right\}_{i \in \mathbb{N}}$, where $p_{i} \in(0,1)$, in such a way that

$$
\prod_{i \in \mathbb{N}} p_{i}^{\prod_{j=0}^{i} N_{j}}>0
$$

(or, equivalently, $\sum_{i \in \mathbb{N}}\left(1-p_{i}\right) \prod_{j=0}^{i} N_{j}<\infty$ ). Using (4.2), if we condition on $\mathcal{A}$, the probability that every particle places its children (if any) to its right is bounded from below by $\prod_{i \in \mathbb{N}} p_{i}^{\prod_{j=0}^{i} N_{j}}$. This implies that there is a positive probability of global, nonlocal survival.

We choose the sequences $\left\{N_{i}\right\}_{i \in \mathbb{N}}$ and $\left\{p_{i}\right\}_{i \in \mathbb{N}}$ to respectively satisfy (4.1) and (4.2) as follows. Choose a sequence $\left\{\alpha_{i}\right\}_{i \in \mathbb{N}}$ such that $\alpha_{i} \in(0,1)$ for all $i \in \mathbb{N}$ and $\prod_{i \in \mathbb{N}} \alpha_{i}>1-\bar{q}$. Then, iteratively, if we fixed $N_{0}, \ldots, N_{k}$, since $\lim _{x \rightarrow \infty} \rho([0, x])=1$, there exists $N_{k+1} \in \mathbb{N}$ such that $\rho\left(\left[0, N_{k+1}\right]\right)>\alpha_{k+1}^{1 / \prod_{j=0}^{k} N_{j}}$. Let us take, for instance, $p_{i}>1 /\left(i \prod_{j=0}^{i} N_{j}\right)$.

We note that the class constructed in this example includes discrete-time counterparts of continuous-time BRWs where $\rho$ can be chosen as in (2.3), where $k(x) \equiv k$ does not depend on $x, k_{x y}:=k p(x, y)$ (where $P$ is defined as before), and $\lambda>\lambda_{s}$ is fixed. Finally, we observe that this example extends naturally to an example of a site-breeding BRW on a radial tree where the number of branches of a vertex at distance $k$ from the root is at least $1 / p(k, k+1)$.

Even though the local extinction probability $\boldsymbol{q}(\cdot, y)$ (for any fixed $y \in \mathbb{N}$ ) of Examples 4.4 and 4.5 provides a fixed point which is different from both $\overline{\boldsymbol{q}}$ and $\mathbf{1}$ for the function $G$ of an irreducible BRW, in the following example we give a more explicit construction of such a fixed point. 
Remark 4.1. Consider a generating function

$$
G(z \mid n)= \begin{cases}\frac{3\left(p_{n} z(n+1)+\left(1-p_{n}\right) z(n-1)\right)^{2}}{4}+\frac{1}{4}, & n \geq 1, \\ \frac{3 z(1)^{2}}{4}+\frac{1}{4}, & n=0,\end{cases}
$$

on $[0,1]^{\mathbb{N}}$, where $p_{n} \in(0,1)$ for all $n \in \mathbb{N}$. This is the generating function of an irreducible BRW, and the constant vectors $\overline{\boldsymbol{q}} \equiv \frac{1}{3}$ and $\mathbf{1}$ are always fixed points of $G$ regardless of the choice of $\left\{p_{n}\right\}$. An explicit construction of $\left\{p_{n}\right\}$ and of a third fixed point $z \in(0,1)^{X}$ can be carried out recursively as follows. Take $z(0) \in\left(\frac{1}{3}, 1\right), p_{0}=1$, and $p_{1}<\frac{5}{9}$. The explicit expression of the equation $G(z)=z$ is easily derived from (4.3). Since $1>\sqrt{(4 x-1) / 3}>x$ for all $x \in\left(\frac{1}{3}, 1\right)$, then $1>z(1)>z(0)>\frac{1}{3}$. Suppose that $1>\boldsymbol{z}(n)>\boldsymbol{z}(n-1)>\cdots>\boldsymbol{z}(0)>\frac{1}{3}$ for some $p_{0}, p_{1}, \ldots, p_{n-1} \in(0,1)$. Choose $z(n+1)<1$ such that $3 z(n+1)^{2} / 4+\frac{1}{4}>z(n)$. By continuity, there exists $p_{n}<1$ such that $3\left(p_{n} z(n+1)+\left(1-p_{n}\right) z(n-1)\right)^{2} / 4+\frac{1}{4}=z(n)$. By induction we have a new fixed point $z$ of this $G$ (associated to the sequence $\left\{p_{n}\right\}$ ) such that $\frac{1}{3}<z(n)<z(n+1)<1$ for all $n$ and $\lim _{n \rightarrow \infty} p_{n}=\lim _{n \rightarrow \infty} z(n)=1^{-}$.

The above disproves [25, Theorem 3 and Corollary 4]. Indeed, there is an error in the proof of [25, Theorem 3]. In line 8 of the proof, the sentence 'Clearly $B^{\prime} \neq \varnothing$ ' is incorrect when the set $X$ is infinite as the following example shows. Using the notation of [25], take $\boldsymbol{x}, \boldsymbol{t} \in[0,1]^{X}$ such that $\boldsymbol{x}$ is constant, say $\boldsymbol{x}(i)=a<1$ for all $i \in X$ and $1>\boldsymbol{t}(i)>a$ for all $i \in X$. Suppose that $\sup _{i \in X} \boldsymbol{t}(i)=1$. Then the half-line $\{\boldsymbol{x}+\theta(\boldsymbol{t}-\boldsymbol{x}): \theta \geq 0\}$ exits from the set $[0,1]^{X}$ at $\theta=1$, that is, at the point $t$. Indeed, if $\theta>1$ then $\sup _{i \in X}(\boldsymbol{x}(i)+$ $\theta(\boldsymbol{t}(i)-\boldsymbol{x}(i)))=\theta \sup _{i \in X} \boldsymbol{t}(i)-a(\theta-1)=\theta-a(\theta-1)=1+(\theta-1)(1-a)>1$. But $B^{\prime}:=\{i \in X: t(i)=1\}=\varnothing$; roughly speaking, in this case there is not a smallest value for $\theta \geq 0$ such that some coordinates of the point $\boldsymbol{x}+\theta(\boldsymbol{t}-\boldsymbol{x})$ are 1 .

\section{Proofs}

Proof of Proposition 2.1. If $\overline{\boldsymbol{q}}=\mathbf{1}$, there is nothing to prove. Suppose that $\overline{\boldsymbol{q}}<\mathbf{1}$. Without loss of generality we can suppose that $\overline{\boldsymbol{q}}(x)<1$ for all $x \in X$. Indeed, given $x_{0}$ such that $\overline{\boldsymbol{q}}\left(x_{0}\right)=1$, then, for all $x \in \mathcal{N}_{x_{0}}$, we have $\overline{\boldsymbol{q}}(x)=1$. Since we defined $\widehat{\boldsymbol{z}}(x):=1$ whenever $\overline{\boldsymbol{q}}(x)=1$, we can remove these vertices to obtain a new set $X^{\prime} \subseteq X$. Consider the restricted BRW on $X^{\prime}$ (obtained by killing all the particles going outside $\bar{X}^{\prime}$ ). It is clear that $\boldsymbol{q}^{X}(x, A) \leq \boldsymbol{q}^{X^{\prime}}(x, A)$ for all $x \in X^{\prime}$ and $A \subseteq X^{\prime}$. The generating function $G^{\prime}$ of the new BRW satisfies $G^{\prime}\left(\left(z_{\mid X^{\prime}}\right) \mid x\right) \geq G(z \mid x)$ for all $x \in X^{\prime}$; hence, $G(z) \geq z$ implies that $G^{\prime}\left(z_{\mid X^{\prime}}\right) \geq \boldsymbol{z}_{\mid X^{\prime}}$ (where $z_{\mid X^{\prime}}$ is $z$ restricted to $X^{\prime}$ ). Moreover, $\widehat{z}$ satisfies the conclusions of the proposition if and only if $\widehat{z_{\mid X^{\prime}}} \equiv \widehat{\boldsymbol{z}}_{\mid X^{\prime}}$ does. Thus, it is enough to prove the result for the BRW restricted to $X^{\prime}$.

Note that $\widehat{z}:=T_{\bar{q}}^{-1}(z)$; thus, $G(z) \geq z$ is equivalent to $\widehat{G}(\widehat{z}) \geq \widehat{z}$. Hence, it is enough to prove the proposition when $\mu_{x}(\mathbf{0})=0$ for all $x \in X$, which implies that $\overline{\boldsymbol{q}}=\mathbf{0}$ and $\widehat{\boldsymbol{z}}=z$. Suppose that $\mathcal{N}_{x}$ is nonempty, $\boldsymbol{z}(y) \leq \boldsymbol{z}(x)$ for all $y \in \mathcal{N}_{x}$, and $\boldsymbol{z}\left(y_{0}\right)<\boldsymbol{z}(x)$ for some $y_{0} \in \mathcal{N}_{x}$. Then, using the facts that $z \leq \mathbf{1}$ and $\prod_{y \in X} z(y)^{f(y)} \leq \boldsymbol{z}(x)$ if $\mathscr{H}(f) \geq 1$, it follows that $z(x) \leq G(z \mid x) \leq \sum_{\left\{f \in S_{X}: f\left(y_{0}\right)=0\right\}} \mu_{x}(f) z(x)+\sum_{\left\{f \in S_{X}: f\left(y_{0}\right)>0\right\}} \mu_{x}(f) z\left(y_{0}\right)<z(x)$, which is a contradiction. If in particular $\mathbf{z}(x)=1$, since $z(y) \leq 1=z(x)$ for all $y \in X$ then we have $z(y)=1$ for all $y \in \mathcal{N}_{x}$. Finally, by induction, we obtain the result for the set $\{y \in X: x \rightarrow y\}$.

Proof of Theorem 3.3. Indeed, since $\left\{\boldsymbol{q}_{n}(\cdot, A)\right\}_{n \in \mathbb{N}}$ is nondecreasing,

$$
\boldsymbol{q}_{n}(\cdot, A)=G\left(\boldsymbol{q}_{n-1}(\cdot, A)\right),
$$


and $\overline{\boldsymbol{q}}$ is the smallest fixed point of $G$, we immediately have

$$
\boldsymbol{q}(\cdot, A)=\overline{\boldsymbol{q}}(\cdot) \quad \Longleftrightarrow \quad \boldsymbol{q}_{0}(\cdot, A) \leq \overline{\boldsymbol{q}}(\cdot)
$$

that is, the equivalence (a) $\Leftrightarrow$ (b) holds. Moreover, the event 'local survival in $A$ starting from $x$ ' implies both 'global survival starting from $x$ ' and 'visiting $A$ at least once starting from $x$ '; hence, $\boldsymbol{q}(x, A)=\overline{\boldsymbol{q}}(x)<1$ if and only if the probability of visiting $A$ infinitely many times starting from $x$ conditioned on global survival is 1 and (a) $\Leftrightarrow(e) \Rightarrow$ (d). Trivially, (b) $\Leftrightarrow$ (c) and (d) $\Rightarrow$ (c). This proves the equivalence.

Before proving Corollary 3.2 and Theorem 3.4, we need two lemmas.

Lemma 5.1. Let $(X, \mu)$ be a BRW, and fix $z, v \in[0,1]^{X}$ such that $z+\varepsilon v \in[0,1]^{X}$ for some $\varepsilon>0$. Then the function $t \mapsto G(z+v t \mid x)$ is strictly convex if and only if there exists

$$
f: \mu_{x}(f)>0, \quad \sum_{y \in \operatorname{supp}(v)} f(y) \geq 2, \quad \operatorname{supp}(z) \cup \operatorname{supp}(v) \supseteq \operatorname{supp}(f) .
$$

Proof. Let us evaluate the function $G$ on the line $t \mapsto z+t v$, where $t \in[0, T)$ and $T:=\sup \left\{s>0: z+s v \in[0,1]^{X}\right\}$ :

$$
\begin{aligned}
& G(z+t v \mid x)=\sum_{f \in S_{X}} \mu_{x}(f) \prod_{y \in X} \sum_{i=0}^{f(y)}\left(\begin{array}{c}
f(y) \\
i
\end{array}\right) z(y)^{f(y)-i} v(y)^{i} t^{i} \\
& =\sum_{f \in S_{X}} \mu_{x}(f) \sum_{\left\{g \in S_{X}: g \leq f\right\}} \prod_{y \in X}\left(\begin{array}{l}
f(y) \\
g(y)
\end{array}\right) z(y)^{f(y)-g(y)} v(y)^{g(y)} t^{g(y)} \\
& =\sum_{f \in S_{X}} \mu_{x}(f) \sum_{\left\{g \in S_{X}: g \leq f\right\}} t^{\mathcal{H}(g)} \prod_{y \in X}\left(\begin{array}{l}
f(y) \\
g(y)
\end{array}\right) z(y)^{f(y)-g(y)} v(y)^{g(y)} \\
& =\sum_{f \in S_{X}} \mu_{X}(f) \sum_{i=0}^{\infty} \sum_{\left\{g \in S_{X}: \mathcal{H}(g)=i, g \leq f\right\}} t^{i} \prod_{y \in X}\left(\begin{array}{c}
f(y) \\
g(y)
\end{array}\right) z(y)^{f(y)-g(y)} v(y)^{g(y)} \\
& =\sum_{i=0}^{\infty} t^{i}\left(\sum_{\left\{f, g \in S_{X}: \mathscr{H}(g)=i, g \leq f\right\}} \mu_{x}(f) \prod_{y \in X}\left(\begin{array}{c}
f(y) \\
g(y)
\end{array}\right) z(y)^{f(y)-g(y)} v(y)^{g(y)}\right) .
\end{aligned}
$$

The strict convexity of a power series in $t$ with nonnegative coefficients is equivalent to the strict positivity of at least one coefficient corresponding to $t^{i}$ with $i \geq 2$. Hence, it is easy to show that each of the following assertions is equivalent to the next and that they are all equivalent to the strict convexity of $t \mapsto G(z+v t \mid x)$.

(i) There exists $f, g: \mathscr{H}(g) \geq 2, f \geq g, \mu_{x}(f)>0, \operatorname{supp}(v) \supseteq \operatorname{supp}(g), \operatorname{supp}(z) \supseteq$ $\operatorname{supp}(f-g)$.

(ii) There exists $f, g: \mathscr{H}(g) \geq 2, f \geq g, \mu_{x}(f)>0, g=f \mathbf{1}_{\operatorname{supp}(v)}, \operatorname{supp}(z) \supseteq \operatorname{supp}(f) \backslash$ $\operatorname{supp}(v)$.

(iii) There exists $f: \mu_{x}(f)>0, \sum_{y \in \operatorname{supp}(v)} f(y) \geq 2, \operatorname{supp}(z) \supseteq \operatorname{supp}(f) \backslash \operatorname{supp}(v)$.

(iv) There exists $f: \mu_{x}(f)>0, \sum_{y \in \operatorname{supp}(v)} f(y) \geq 2, \operatorname{supp}(z) \cup \operatorname{supp}(v) \supseteq \operatorname{supp}(f)$. 
Lemma 5.2. Let $(X, \mu)$ be a BRW, and fix $x_{0} \in X$. Suppose that, for some $\bar{x}$ in the same irreducible class of $x_{0}$ and $f \in S_{X}$, we have $\mu_{\bar{x}}(f)>0, \sum_{\left\{w: w \rightleftharpoons x_{0}\right\}} f(w) \geq 2$. We can fix $\bar{n} \in \mathbb{N}$ such that if the process starts with one particle at $x_{0} \in X$ then we have at least two particles at $x_{0}$ in the generation $\bar{n}$ w.p.p.

Proof. Consider a path $x_{0}, x_{1}, \ldots, x_{m}=\bar{x}$, and let $f \in S_{X}$ be such that $\mu_{\bar{x}}(f)>0$ and $\sum_{\left\{w: w \rightleftharpoons x_{0}\right\}} f(w) \geq 2$. We have the following two cases.

Case 1. There exists $x_{m+1} \in X$ such that $x_{m+1} \rightleftharpoons x_{0}$ and $f\left(x_{m+1}\right) \geq 2$; in this case consider the closed path $x_{0}, x_{1}, x_{2}, \ldots, x_{m}, x_{m+1}, \ldots, x_{n}=x_{0}$ and take $\bar{n}:=n$. Since any particle at $x_{i}$ has at least one child at $x_{i+1}$ w.p.p., and a particle at $\bar{x}$ has at least two children at $x_{m+1}$ w.p.p., then any particle at $x_{0}$ has at least two descendants at $x_{0}$ in the $\bar{n}$ th generation w.p.p. Indeed, let $f_{i} \in S_{X}$ such that $\mu_{x_{i}}\left(f_{i}\right)>0, f_{i}\left(x_{i+1}\right) \geq 1$ for all $i=0, \ldots \bar{n}-1\left(f_{m}\right.$ being $\left.f\right)$. Then the probability that a particle at $x_{0}$ has at least two descendants at $x_{0}$ in the $\bar{n}$ th generation is bounded from below by $\prod_{i=0}^{m} \mu_{i}\left(f_{i}\right) \prod_{j=m+1}^{\bar{n}-1} \mu_{j}\left(f_{i}\right)^{2}$.

Case 2. There exists a couple of different vertices $x_{m+1}, y_{m+1}$ such that $x_{m+1}, y_{m+1} \rightleftharpoons x_{0}$ and $f\left(x_{m+1}\right), f\left(y_{m+1}\right) \geq 1$; in this case, consider the paths $x_{0}, x_{1}, \ldots x_{m}, x_{m+1}, \ldots, x_{n_{1}}=x_{0}$ and $x_{0}, x_{1}, \ldots x_{m}, y_{m+1}, \ldots, y_{n_{2}}=x_{0}$, and take $\bar{n}:=\operatorname{LCM}\left(n_{1}, n_{2}\right)$ (the conclusion follows as before).

Proof of Theorem 3.4. (a) For every fixed point $z$ of $G$, we know that $z \geq \overline{\boldsymbol{q}}$ and $z \leq \mathbf{1}_{X}$. This implies that if $\sup _{x \in X} z(x)<1$ for some fixed point then, necessarily, $\sup _{x \in X} \overline{\boldsymbol{q}}(x)<1$. Hence, if $\overline{\boldsymbol{q}}=\mathbf{1}$, there is nothing to prove. Otherwise, we show that if $G(\boldsymbol{z})=\boldsymbol{z}$ and $\boldsymbol{z} \neq \overline{\boldsymbol{q}}$, then $\sup _{w \in X} z(w)=1$. Suppose that the BRW is locally isomorphic to $(Y, v)$ through the map $g$ and define $\boldsymbol{h}(y):=\sup _{w \in g^{-1}(y)} \boldsymbol{z}(w)$. Clearly, $\boldsymbol{h} \in[0,1]^{Y}$ and $\boldsymbol{h} \circ g \geq \boldsymbol{z}$, which implies that $G_{Y}(\boldsymbol{h}) \geq \boldsymbol{h}$. Indeed,

$$
\begin{aligned}
G_{Y}(\boldsymbol{h} \mid y) & =\sup _{x \in g^{-1}(y)} G_{Y}(\boldsymbol{h} \mid g(x)) \\
& =\sup _{x \in g^{-1}(y)} G(\boldsymbol{h} \circ g \mid x) \\
& \geq \sup _{x \in g^{-1}(y)} G(z \mid x) \\
& =\sup _{x \in g^{-1}(y)} z(x) \\
& =\boldsymbol{h}(y) .
\end{aligned}
$$

If $Y$ finite then we can choose $\tilde{y} \in Y$ which minimizes

$$
t(y):=\frac{1-\overline{\boldsymbol{q}}^{Y}(y)}{\boldsymbol{h}(y)-\overline{\boldsymbol{q}}^{Y}(y)}
$$

(where $t(y):=+\infty$ if $\boldsymbol{h}(y)=\overline{\boldsymbol{q}}^{Y}(y)$ ). Note that $t(y) \geq 1$ for all $y \in Y$ and $t(\tilde{y})<+\infty$. By applying the maximum principle (Proposition 2.1) to the function $1 / t(y)$ (where $y$ is ranging in the set $\left.\left\{w: \overline{\boldsymbol{q}}^{Y}(w)<1\right\}\right)$, it follows that it is constant on $\{y: \tilde{y} \rightarrow y\}$. Since $\overline{\boldsymbol{q}}^{Y}(\widetilde{y})<1$ and $Y$ is finite, then there exists $y_{0}$ such that $\tilde{y} \rightarrow y_{0}$ and there is local survival at $y_{0}$ starting from $y_{0}$. Since $(Y, v)$ satisfies Assumption 2.1, then there exists $\bar{y} \rightleftharpoons y_{0}$ such that a particle living at $\bar{y}$ w.p.p. has at least two children in the irreducible class of $y_{0}$. Then, by taking $y_{0}$ instead of $x_{0}$ in Lemma 5.2, we can find $\bar{n} \in \mathbb{N}$ such that the function

$$
\phi(t):=G_{Y}^{(\bar{n})}\left(\overline{\boldsymbol{q}}^{Y}+t\left(\boldsymbol{h}-\overline{\boldsymbol{q}}^{Y}\right) \mid y_{0}\right)-\overline{\boldsymbol{q}}^{Y}\left(y_{0}\right)-t\left(\boldsymbol{h}\left(y_{0}\right)-\overline{\boldsymbol{q}}^{Y}\left(y_{0}\right)\right)
$$


is strictly convex by Lemma 5.1. Indeed, $G_{Y}^{(\bar{n})}$ is the generating function of the BRW constructed by considering the $n$th generations of the original BRW, where $\bar{n} \mid n$ and, under our hypotheses, it satisfies (5.1).

Note that $\phi$ is well defined in $\left[0, t\left(y_{0}\right)\right]$ since

$$
\boldsymbol{r}_{t}(y):=\overline{\boldsymbol{q}}^{Y}(y)+t\left(\boldsymbol{h}(y)-\overline{\boldsymbol{q}}^{Y}(y)\right) \leq \overline{\boldsymbol{q}}^{Y}(y)+t\left(y_{0}\right)\left(\boldsymbol{h}(y)-\overline{\boldsymbol{q}}^{Y}(y)\right) \leq 1 .
$$

Hence, $\boldsymbol{r}_{t} \in[0,1]^{Y}$ for all $t \in\left[0, t\left(y_{0}\right)\right]$.

Clearly, every fixed point of $G_{Y}$ is a fixed point of $G_{Y}^{(\bar{n})}$; in particular, $G^{(\bar{n})}(z)=z$ and $G_{Y}^{(\bar{n})}\left(\overline{\boldsymbol{q}}^{Y}\right)=\overline{\boldsymbol{q}}^{Y}$, whence $\phi(0)=0$ and $\phi(1)=G_{Y}^{(\bar{n})}\left(\boldsymbol{h} \mid y_{0}\right)-\boldsymbol{h}\left(y_{0}\right)$. Now, using (2.6), $G_{Y}^{(\bar{n})}(\boldsymbol{h}) \geq \boldsymbol{h}$ and this, in turn, implies that $\phi(1) \geq 0$. Since $\phi$ is strictly convex, $\phi(t)>0$ for all $t \in\left(1, t\left(y_{0}\right)\right]$. If $t\left(y_{0}\right)>1$ then $0<\phi\left(t\left(y_{0}\right)\right)=G_{Y}^{(\bar{n})}\left(\boldsymbol{r}_{t\left(y_{0}\right)} \mid y_{0}\right)-1$, but this is a contradiction since $\boldsymbol{r}_{t\left(y_{0}\right)} \in[0,1]^{Y}$ and $G_{Y}^{(\bar{n})}\left(\boldsymbol{r}_{t\left(y_{0}\right)}\right) \in[0,1]^{Y}$. In the end $t\left(y_{0}\right)=1$; thus, $1=\boldsymbol{h}\left(y_{0}\right)=\sup _{w \in X} z(w)$.

(b) This applies to $z(\cdot)=\boldsymbol{q}(\cdot, x)$ for any fixed $x \in X$. If the BRW is irreducible, $\boldsymbol{q}(w, x)=$ $\boldsymbol{q}(w, y)$ for all $x, y, w \in X$. Thus, there exists $x \in X$ such that $\boldsymbol{q}(\cdot, x)=\overline{\boldsymbol{q}}(\cdot)$ if and only if $\boldsymbol{q}(w, w)=\overline{\boldsymbol{q}}(w)$ for all $w \in X$; analogously, there exists $x \in X$ such that $\sup _{w \in X} \boldsymbol{q}(w, x)=1$ if and only if $\sup _{w \in X} \boldsymbol{q}(w, w)=1$.

Note that, from the first part of the previous proof, if the BRW on $Y$ is irreducible, then, by the maximum principle, $\left(\boldsymbol{h}-\overline{\boldsymbol{q}}^{Y}\right) /\left(\mathbf{1}-\overline{\boldsymbol{q}}^{Y}\right)$ is a constant function. Thus,

$$
\boldsymbol{h}(y)=\sup _{w \in g^{-1}(y)} \boldsymbol{z}(w)=1 \quad \text { for all } y \in Y .
$$

Proof of Corollary 3.1. When $X$ is finite, $(X, \mu)$ is clearly an $\mathcal{F}$-BRW. If $\overline{\boldsymbol{q}}=\mathbf{1}$, there is nothing to prove. Suppose that $\overline{\boldsymbol{q}}<\mathbf{1}$. Since the BRW is irreducible, $\overline{\boldsymbol{q}}(x)<1$ for all $x \in X$. Let $\overline{\boldsymbol{q}}<\boldsymbol{z}<\mathbf{1}$ be a solution of $G(\boldsymbol{z}) \geq \boldsymbol{z}$. Since $X$ is finite and $\overline{\boldsymbol{q}}<\boldsymbol{z}$ from Theorem 3.4(a), we have $z(x)=1$ for some $x \in X$. By Proposition 2.1, using irreducibility, $\widehat{z}=\mathbf{1}$, which contradicts $z<\mathbf{1}$.

Proof of Corollary 3.2. Since $\left(X, E_{\mu}\right)$ is irreducible, $\boldsymbol{q}(x, y)=\boldsymbol{q}(x, x)$ for all $x, y \in X$, and if $\overline{\boldsymbol{q}}<\mathbf{1}$ (respectively $\boldsymbol{q}(\cdot, y)<\mathbf{1}$ ) then $\overline{\boldsymbol{q}}(x)<1$ (respectively $\boldsymbol{q}(x, y)<1$ ) for all $x \in X$. Moreover, quasitransitivity implies that if $\boldsymbol{q}(\cdot, y)<\mathbf{1}$ then $\sup _{x \in X} \boldsymbol{q}(x, y)<1$. Thus, according to Theorem $3.4, \boldsymbol{q}(\cdot, y) \neq \mathbf{1}$ implies that $\boldsymbol{q}(\cdot, y)=\overline{\boldsymbol{q}}$.

\section{Acknowledgement}

The authors are grateful to the anonymous referee for carefully reading the manuscript and for useful suggestions which helped to improve the paper.

\section{References}

[1] Athreya, K. B. And Ney, P. E. (1972). Branching Processes. Springer, New York.

[2] Belhadj, L. and Lanchier, N. (2006). Individual versus cluster recoveries within a spatially structured population. Ann. Appl. Prob. 16, 403-422.

[3] Belhadji, L., Bertacchi, D. And Zucca, F. (2010). A self-regulating and patch subdivided population. $A d v$. Appl. Prob. 42, 899-912.

[4] Bertacchi, D., Lanchier, N. and Zucca, F. (2011). Contact and voter processes on the infinite percolation cluster as models of host-symbiont interactions. Ann. Appl. Prob. 21, 1215-1252.

[5] Bertacchi, D., Posta, G. And Zucca, F. (2007). Ecological equilibrium for restrained branching random walks. Ann. Appl. Prob. 17, 1117-1137. 
[6] Bertacchi, D. ANd Zucca, F. (2008). Critical behaviors and critical values of branching random walks on multigraphs. J. Appl. Prob. 45, 481-497.

[7] Bertacchi, D. AND ZuCCA, F. (2009). Approximating critical parameters of branching random walks. J. Appl. Prob. 46, 463-478.

[8] Bertacchi, D. and Zucca, F. (2009). Characterization of critical values of branching random walks on weighted graphs through infinite-type branching processes. J. Statist. Phys. 134, 53-65.

[9] Bertacchi, D. AND ZuCCA, F. (2012). Recent results on branching random walks. In Statistical Mechanics and Random Walks: Principles, Processes and Applications, Nova Science Publishers, pp. 289-340.

[10] Biggins, J. D. (1977). Martingale convergence in the branching random walk. J. Appl. Prob. 14, $25-37$.

[11] Biggins, J. D. (1978). The asymptotic shape of the branching random walk. Adv. Appl. Prob. 10, 62-84.

[12] Biggins, J. D. and Kyprianou, A. E. (1997). Seneta-Heyde norming in the branching random walk. Ann. Prob. 25, 337-360.

[13] Biggins, J. D. and Rahimzadeh Sani, A. (2005). Convergence results on multitype, multivariate branching random walks. Adv. Appl. Prob. 37, 681-705.

[14] Galton, F. and Watson, H.W. (1875). On the probability of the extinction of families. J. Anthropological Inst. Great Britain Ireland 4, 138-144.

[15] Gantert, N., Müller, S., Popov, S. and Vachkovskaia, M. (2010). Survival of branching random walks in random environment. J. Theoret. Prob. 23, 1002-1014.

[16] Harris, T. E. (1963). The Theory of Branching Processes. Springer, Berlin.

[17] Hueter, I. ANd Lalley, S.P. (2000). Anisotropic branching random walks on homogeneous trees. Prob. Theory Relat. Fields 116, 57-88.

[18] Liggett, T. M. (1996). Branching random walks and contact processes on homogeneous trees. Prob. Theory Relat. Fields 106, 495-519.

[19] Liggett, T. M. (1999). Branching random walks on finite trees. In Perplexing Problems in Probability: Festschrift in Honor of Harry Keston (Progr. Prob. 44), Birkhäuser, Boston, MA.

[20] Madras, N. And Schinazi, R. (1992). Branching random walks on trees. Stoch. Proc. Appl. 42, $255-267$.

[21] Menshikov, M. V. And Volkov, S. E. (1997). Branching Markov chains: qualitative characteristics. Markov Proc. Relat. Fields 3, 225-241.

[22] Mountford, T. And Schinazi, R. B. (2005). A note on branching random walks on finite sets. J. Appl. Prob. 42, 287-294.

[23] Müller, S. (2008). Recurrence for branching Markov chains. Electron. Commun. Prob. 13, (2008), 576-605.

[24] Pemantle, R. and Stacey, A. M. (2001). The branching random walk and contact process on Galton-Watson and nonhomogeneous trees. Ann. Prob. 29, 1563-1590.

[25] Spataru, A. (1989). Properties of branching processes with denumerably many types. Rev. Roumaine Math. Pures Appl. 34, 747-759.

[26] Stacey, A. (2003). Branching random walks on quasi-transitive graphs. Combinatorics Prob. Comput. 12, 345-358.

[27] Woess, W. (2000). Random walks on infinite graphs and groups. (Cambr. Tracts Math. 138), Cambridge University Press.

[28] Zucca, F. (2011). Survival, extinction and approximation of discrete-time branching random walks. J. Statist. Phys. 142, 726-753. 